\title{
TAK1-mediated autophagy and fatty acid oxidation prevent hepatosteatosis and tumorigenesis
}

\author{
Sayaka Inokuchi-Shimizu, ${ }^{1}$ Eek Joong Park, ${ }^{1}$ Yoon Seok Roh, ${ }^{1}$ Ling Yang, ${ }^{1,2}$ Bi Zhang, ${ }^{1}$ Jingyi Song,, Shuang Liang, ${ }^{1}$ \\ Michael Pimienta, ${ }^{1}$ Koji Taniguchi, ${ }^{3}$ Xuefeng Wu, ${ }^{3}$ Kinji Asahina, ${ }^{4}$ William Lagakos, ${ }^{1}$ Mason R. Mackey, ${ }^{5}$ Shizuo Akira, ${ }^{6}$ \\ Mark H. Ellisman, ${ }^{5}$ Dorothy D. Sears, ${ }^{1}$ Jerrold M. Olefsky, ${ }^{1}$ Michael Karin, ${ }^{3}$ David A. Brenner, ${ }^{1}$ and Ekihiro Seki ${ }^{1}$ \\ 'Department of Medicine, UCSD, School of Medicine, La Jolla, California, USA. ²Division of Castroenterology, Department of Internal Medicine, Union Hospital, Tongji Medical College, Huazhong University \\ of Science and Technology, Wuhan, China. ${ }^{3}$ Laboratory of Gene Regulation and Signal Transduction, Departments of Pharmacology and Pathology, UCSD, School of Medicine, La Jolla, California, USA. \\ ${ }^{4}$ Department of Pathology, University of Southern California Keck School of Medicine, Los Angeles, California, USA. ${ }^{5}$ National Center for Microscopy and Imaging Research and Department \\ of Neurosciences, UCSD, School of Medicine, La Jolla, California, USA. 'Laboratory of Host Defense, WPI Immunology Frontier Research Center, Osaka University, Suita, Osaka, Japan.
}

\begin{abstract}
The MAP kinase kinase kinase TCF $\beta$-activated kinase 1 (TAK1) is activated by TLRs, IL-1, TNF, and TGF $\beta$ and in turn activates IKK-NF-кB and JNK, which regulate cell survival, growth, tumorigenesis, and metabolism. TAK1 signaling also upregulates AMPK activity and autophagy. Here, we investigated TAK1-dependent regulation of autophagy, lipid metabolism, and tumorigenesis in the liver. Fasted mice with hepatocyte-specific deletion of Tak1 exhibited severe hepatosteatosis with increased mTORC1 activity and suppression of autophagy compared with their WT counterparts. TAK1-deficient hepatocytes exhibited suppressed AMPK activity and autophagy in response to starvation or metformin treatment; however, ectopic activation of AMPK restored autophagy in these cells. Peroxisome proliferator-activated receptor $\alpha$ (PPAR $\alpha$ ) target genes and $\beta$-oxidation, which regulate hepatic lipid degradation, were also suppressed in hepatocytes lacking TAK1. Due to suppression of autophagy and $\beta$-oxidation, a high-fat diet challenge aggravated steatohepatitis in mice with hepatocyte-specific deletion of Tak1. Notably, inhibition of mTORC1 restored autophagy and PPAR $\alpha$ target gene expression in TAK1-deficient livers, indicating that TAK1 acts upstream of mTORC1. mTORC1 inhibition also suppressed spontaneous liver fibrosis and hepatocarcinogenesis in animals with hepatocyte-specific deletion of Tak1. These data indicate that TAK1 regulates hepatic lipid metabolism and tumorigenesis via the AMPK/mTORC1 axis, affecting both autophagy and PPAR $\alpha$ activity.
\end{abstract}

\section{Introduction}

TGF $\beta$-activated kinase 1 (TAK1) is a MAP kinase kinase kinase (MAP3K) activated by TLRs, IL-1 receptor, TNF receptor 1 , and TGF $\beta$ receptors $(1,2)$. In hepatocytes, TAK1 stimulates activation of IKK-NF- $\kappa \mathrm{B}$ and JNK, which are crucial regulators of cell survival, proliferation, and tumorigenesis, as well as lipid metabolism and insulin sensitivity (3). IKK/NF- $\kappa \mathrm{B}$ signaling prevents TNF- and ROS-mediated hepatocyte death, hepatic steatosis, and hepatocarcinogenesis (4). In contrast, sustained JNK activation promotes ROS generation, resulting in hepatocyte death, lipid accumulation, and development of hepatocellular carcinoma (HCC) (3). Since TAK1 controls activation of both IKK/NF- $\mathrm{BB}$ and JNK pathways, its role in liver pathophysiology has been hard to predict. We and others have previously demonstrated that ablation of Tak1 in hepatocytes results in spontaneous hepatocyte death, inflammation, fibrosis, and HCC development; these phenotypes depends on TNF and TGF $\beta$ receptor signaling $(5,6)$ and are more dramatic than the phenotypes observed in mice with hepatocytespecific deletion of IKK $\beta$ or IKK $\gamma / \operatorname{NEMO}(5,7-9)$.

Authorship note: Sayaka Inokuchi-Shimizu, Eek Joong Park, Yoon Seok Roh, and Ling Yang contributed equally to this work.

Conflict of interest: The authors have declared that no conflict of interest exists. Submitted: October 31, 2013; Accepted: May 22, 2014

Reference information: / Clin Invest. 2014;124(8):3566-3578. doi:10.1172/JCl74068.
TAK1 can also regulate AMPK activity through phosphorylation (10). Nutrient deprivation strongly activates AMPK, leading to the inhibition of mTOR complex 1 (mTORC1), a multifunctional protein kinase complex that regulates lipid biosynthesis, cellular proliferation, and autophagy $(11,12)$. AMPK can also stimulate autophagy through direct phosphorylation of ULK1 independently of mTORC1 (13). Under high nutrient conditions, when ATP levels are high, AMPK activity is inhibited, thereby activating mTORC1 and resulting in increased lipid synthesis in a SREBP-1c- and PPAR $\gamma$-dependent manner (12). Moreover, mTORC1 inhibits PPAR $\alpha$ activity, which regulates mitochondrial functions and fatty acid $\beta$-oxidation (FAO) (14). Hepatic FAO is impaired in mice with inactivated PPAR $\alpha$, SIRT1, or SIRT3 (15-17). These mice exhibit significant lipid deposition in the liver upon consuming a high-fat diet (HFD) or during fasting. AMPK activation and mTORC1 inhibition induce autophagy to remove and recycle cellular materials for biosynthesis or energy production when nutrients are limited. Autophagy promotes lipid degradation and prevents excessive lipid accumulation (18). It was suggested that TAK1 might contribute to the induction of autophagy through either the IKK complex or AMPK $(10,19)$. However, the physiological and pathophysiological significance of TAK1-dependent regulation of AMPK/mTORC1 signaling and autophagy and their involvement in lipid metabolism and HCC development in the liver remain elusive. 
In the present study, we determined that TAK1 in hepatocytes prevents excessive lipid accumulation through AMPK activation, mTORC1 inhibition, and autophagy. TAK1 also favors PPAR $\alpha$-mediated FAO through the inhibition of mTORC1. Upon HFD feeding, TAK1 activity prevents excessive hepatic lipid accumulation, injury, and inflammation. Correspondingly, restoration of autophagy with the mTORC1 inhibitor rapamycin suppresses hepatic steatosis, fibrosis, and HCC progression in Alb-Cre Tak1 ${ }^{f / f l}\left(\right.$ Tak1 $\left.1^{\Delta h e p}\right)$ mice.

\section{Results}

Nutrient deprivation causes severe lipid accumulation in Tak1-deleted livers. To determine the physiological role of TAK1 in the liver, we investigated its involvement in the response to fasting, which causes mobilization of lipids from peripheral depots into the liver $(12,20)$. Initially, livers appeared normal in 1-month-old Tak1 $1^{\text {shep }}$ mice and their WT counterparts, but after 12 hours of fasting, livers of Tak $1^{\text {shep }}$ mice became white. Tak $1^{\text {shep }}$ mice showed increased lipid accumulation in hepatocytes compared with that seen in their WT counterparts, particularly after fasting (Figure 1, A and B). Hepatic triglycerides (TGs) in fasted Tak $1^{\text {shep }}$ mice were 3-fold higher than those in fasted WT mice, whereas plasma FFAs were similarly elevated in both fasted Tak $1^{\text {shep }}$ and WT mice (Figure $1, C$ and D). These data indicate that although FFA release from adipose tissue is not affected by loss of hepatic TAK1 expression, the deposition of TGs in the liver is TAK1 dependent. In WT livers, nutrient deprivation strongly inhibited S6 phosphorylation, an established marker of mTORC1 activity (Figure 1E). In contrast, $\mathrm{Tak1}^{-/-}$livers showed marked increases in S6 phosphorylation before or after nutrient deprivation (Figure 1E), suggesting aberrant activation of mTORC1.

AMPK activation and autophagy are inhibited in $\mathrm{Tak1}^{-/-}$hepatocytes. Reduced p62/SQSTM1 expression, increased LC3B-II generation from LC3B-I, and formation of LC3B aggregates are markers of autophagy induction (21-23). We found that fasted WT livers exhibited lower $\mathrm{p} 62$ expression and higher LC3B-II amounts and accumulation of LC3B dots than did fed WT livers (Figure 2, A and B, and Supplemental Figure 1, A and B; supplemental material available online with this article; doi:10.1172/JCI74068DS1). In contrast, $\mathrm{Tak1}^{-/-}$livers exhibited much higher $\mathrm{p} 62$ expression and no LC3B-II expression or less LC3B aggregates than did WT livers (Figure 2, A and B, and Supplemental Figure 1, A and B). Furthermore, fasting did not result in any increase in autophagy markers in $\mathrm{Tak1}^{-/}$livers, indicating that TAK1 is required for stimulation of liver autophagy during fasting. Chloroquine (CQ) treatment prevents autophagosome degradation, thereby inducing LC3B aggregation during fed states (23). In WT livers, CQ treatment increased LC3B aggregation, but LC3B aggregates were much less pronounced in $\mathrm{Tak1}^{-/-}$livers before or after CQ treatment (Supplemental Figure 1F). In LC3B-GFP transgenic mice, the detection of free GFP released from an LC3B-GFP fusion protein by autophagosome degradation reflects autophagic flux (24). We found that free GFP was increased after fasting in WT LC3B-GFP transgenic livers; however, free GFP formation was insignificant in Tak1 $1^{\text {shep }}$ LC3B-GFP transgenic livers before and after fasting (Supplemental Figure 1G). EM showed that there were some lipid droplets with attached autophagic vesicles (AVs) in fed WT livers and that the number of such droplets was increased upon fasting (Figure 2C). However, lipid droplets with attached AVs were rare in Tak1 ${ }^{\Delta h e p}$ livers (Figure 2C). These results demonstrate that both basal and induced autophagy are suppressed in $T a k 1^{-/}$livers. In addition, expression of autophagy genes including Ulk1, Ulk2, Atg3, Atg5, and Atg8/Lc3b was suppressed in Tak $1^{\text {shep }}$ mice, as revealed by microarray analysis and quantitative real-time PCR (Supplemental Table 1 and Supplemental Figure 2), further supporting the requirement of TAK1 for basal and induced autophagy in the liver.

Next, we examined TAK1 kinase activity upon starvation. TAK1 kinase activity was increased 1 hour after starvation (Figure 2D). Starvation also induced the phosphorylation of LKB1, AMPK, raptor, and ULK1 and increased the conversion of LC3B-I to LC3B-II and the accumulation of LC3B-GFP aggregates, while reducing the amount of p62 in WT hepatocytes (Figure 2, E and F). All of these responses were highly attenuated in $\mathrm{Tak1}^{-/-}$hepatocytes (Figure 2, E and F), indicating that TAK1 is required for LKB1 and AMPK phosphorylation and induction of autophagy during starvation. Similarly, we observed that TAK1 kinase activity was increased upon metformin treatment (Supplemental Figure 3A), and metformin-induced AMPK activation and autophagy were suppressed in Tak1/-- hepatocytes (Supplemental Figure 3, B-D). Accordingly, we examined whether ectopic AMPK activation can restore autophagy in $\mathrm{Tak1}^{-/-}$hepatocytes. Overexpression of active AMPK increased LC3B-I-to-LC3B-II conversion and decreased p62 in $\mathrm{Tak1}^{-/-}$hepatocytes, indicating that AMPK is an important downstream effector of TAK1 that mediates its effect on autophagy (Supplemental Figure 3E).

Tak1 deficiency causes defects in PPAR $\alpha$-mediated FAO in hepatocytes. To investigate the role of TAK1 in hepatic lipid metabolism, we assessed expression of genes related to FAO and lipid metabolism. The mRNA levels of Ppara, Hmgcs2, Cpt1a, Acox1, Cyp4a10, and Sirt 3 in fed WT livers were higher than in fed Tak $1^{-/-}$livers (Figure 3A). Furthermore, these mRNAs were upregulated after 12 hours of fasting in WT livers, but their expression in $\mathrm{Tak1}^{-/-}$livers remained lower than in WT livers, even after fasting (Figure 3A). Hepatic FAO was also elevated after fasting in WT mice, and it was suppressed in $\mathrm{Tak1}^{-/}$livers both before and after fasting (Figure 3B). Likewise, WT livers expressed higher levels of $A p o B$ and $M t t p$ mRNA than did Tak1 $1^{-/}$livers both before and after fasting and had higher TG secretion rates (Figure 3, C and D). These results suggest that reduced FAO and impaired TG secretion contribute to increased hepatic steatosis in Tak $1^{\text {shep }}$ mice after fasting.

Because Tak1 deficiency caused overactivation of mTORC1 (Figure 1E) and mTORC1 negatively regulates PPAR $\alpha$ signaling (14), we expected that PPAR $\alpha$ activity would be suppressed in Tak $1^{\text {shep }}$ mice. Microarray transcriptome profiling including gene ontology and KEGG pathway analysis revealed that the PPAR signaling and fatty acid metabolism pathways were in fact the 2 major pathways that were suppressed in the livers of Tak $1^{\Delta h e p}$ mice $\left(P=3.53 \times 10^{-6}\right.$ and $P=2.4 \times 10^{-7}$, respectively) (Supplemental Tables 2 and 3). Forty-eight PPAR $\alpha$ target genes were reported to be involved in FAO and fatty acid metabolism $(16,25,26)$. We found that expression of 28 (after Student's $t$ test) and 14 (after Bonferroni correction) of these genes was significantly decreased in Tak1 $1^{-/}$livers compared with that in WT livers (Supplemental Figure 4 and Supplemental Table 4). This prompted us to further ex- 
A

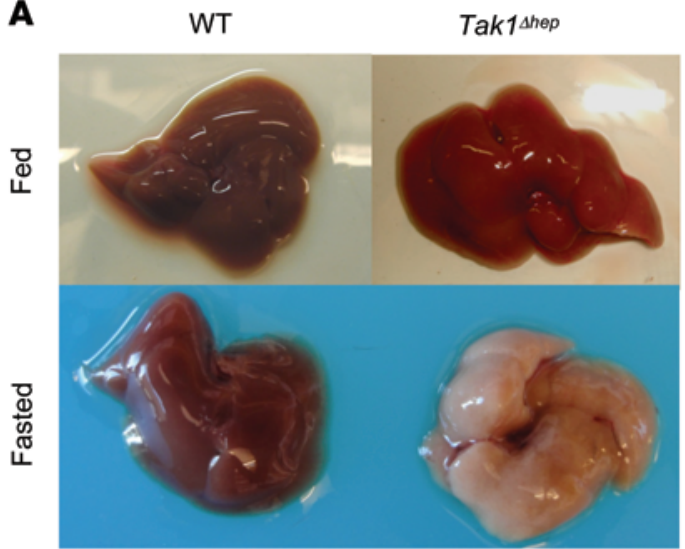

B
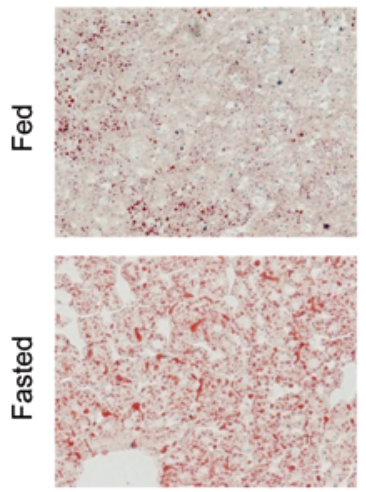
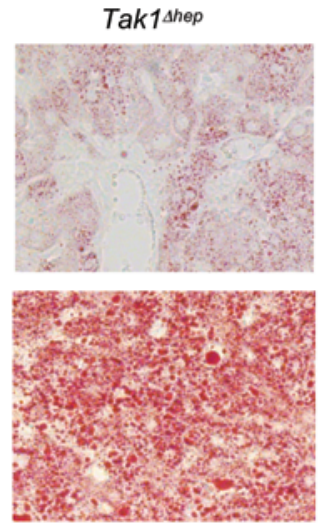

C $\square$ WT $\square$ Tak1thep

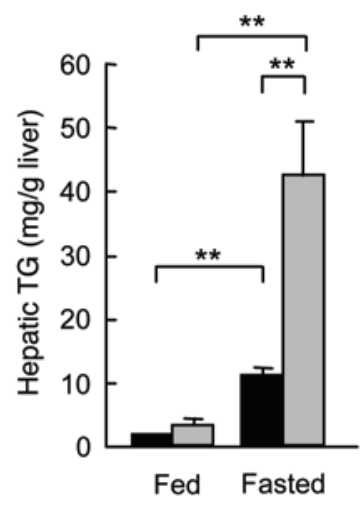

$\square$ WT $\square$ Tak1thep

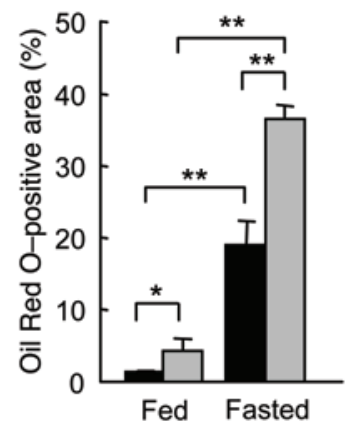

D WT

Tak1thep

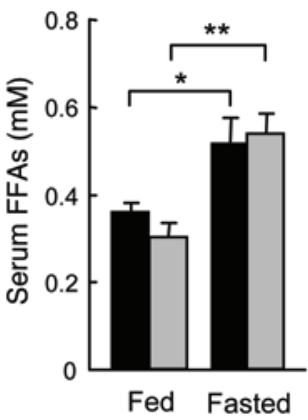

$\mathbf{E}$

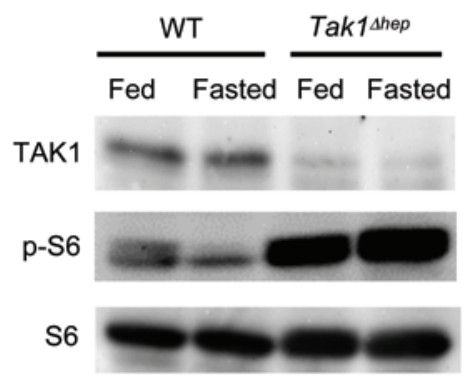

Figure 1. TAK1 deficiency causes severe fatty liver after fasting. One-month-old WT and Tak $1^{\text {shep }}$ mice were fasted for 12 hours ( $n=5$ each). (A) Macroscopic appearance of livers of WT and Tak $7^{\text {hep }}$ mice before and after fasting. (B) Oil Red 0 staining of lipid droplets. Original magnification, $\times 200$. (C) Hepatic TC content and (D) serum levels of FFAs were measured. (E) Immunoblotting for total TAK1, p-S6, and total S6. Black bar, WT; gray bar, Tak ${ }^{\text {1hep }}$ mice. Data are presented as the means \pm SEM. ${ }^{*} P<0.05 ;{ }^{* *} P<0.01$.

amine PPAR $\alpha$-regulated genes using quantitative real-time PCR. Ppara mRNA expression itself was reduced in Tak1 $1^{-/-}$hepatocytes (Figure 4A). Treatment with the PPAR $\alpha$ agonist WY14643 induced Hmgcs2, Acox1, Ehhadh, Acsl1, Peci, Cyp4a10, Cyp4a12, and Cpt1a mRNA in WT hepatocytes, but with the exception of Cpt1a, none of these genes were induced in $\mathrm{Tak1}^{-/-}$hepatocytes (Figure $4 \mathrm{~A})$. We confirmed that the effect of WY14643 on PPAR $\alpha$ target gene expression was mediated through PPAR $\alpha$ and that WY14643 had no significant effect on PPAR $\gamma$ target genes (Supplemental Figure 5). Congruently, we found that WY14643-induced FAO was markedly reduced in Tak1 $1^{-/-}$hepatocytes (Figure 4B). We also examined the effect of WY14643 on palmitate-induced lipid accumulation in hepatocytes. Treatment with palmitate resulted in the accumulation of lipid droplets in WT hepatocytes, and this was strongly enhanced in Tak1 $1^{-/}$hepatocytes (Figure 4C). Interestingly, WY14643 treatment markedly inhibited palmitate-induced lipid accumulation in WT hepatocytes but had no effect in $\mathrm{Tak1}^{-/}$hepatocytes (Figure $4 \mathrm{C}$ ). These results demonstrate that Tak1 deficiency suppresses PPAR $\alpha$ expression and PPAR $\alpha$-induced gene expression, as well as FAO, resulting in enhanced lipid deposition in $\mathrm{Tak1}^{-/-}$hepatocytes.

Rapamycin restores autophagy and lipid degradation in Tak1 $1^{-1}$ hepatocytes. We examined whether inhibition of $\mathrm{mTORC} 1$ with ra- pamycin can restore autophagy in Tak1/- hepatocytes. Autophagy, evidenced by increased LC3B-II GFP generation, accumulation of LC3B GFP aggregates, and reduced p62 accumulation, was induced in both WT and $\mathrm{Tak1}^{-/-}$hepatocytes treated with rapamycin (Figure 5, A and B). Additionally, rapamycin treatment suppressed palmitate-induced lipid accumulation in both WT and $\mathrm{Tak1}^{-1-}$ hepatocytes (Supplemental Figure 6). We examined whether the rapamycin-induced reduction in lipid accumulation is dependent on autophagy. Autophagy inhibition by silencing of Atg5 increased lipid accumulation in both WT and $\mathrm{Tak1}^{-/}$hepatocytes and attenuated the response to rapamycin in both cell types (Supplemental Figure 6). These results suggest that the ability of rapamycin to reduce lipid accumulation in $\mathrm{Tak1}^{-/-}$hepatocytes is largely dependent on autophagy.

Subsequently, we tested the effect of rapamycin in vivo. Rapamaycin suppressed mTORC1 activity in WT and Tak1/-- livers, as shown by decreased S6 phosphorylation (Figure 5C). Consistent with the results of the in vitro experiments, rapamycin treatment induced autophagy in both WT and Tak1/- livers, as demonstrated by decreased p62 and increased LC3B-II and LC3B aggregates (Figure 5, C and D, and Supplemental Figure 1C). Moreover, rapamycin treatment reduced hepatic TG and lipid accumulation in fasted Tak1 $1^{\text {shep }}$ mice (Figure 5, E-G). Rapamycin treatment also in- 
A

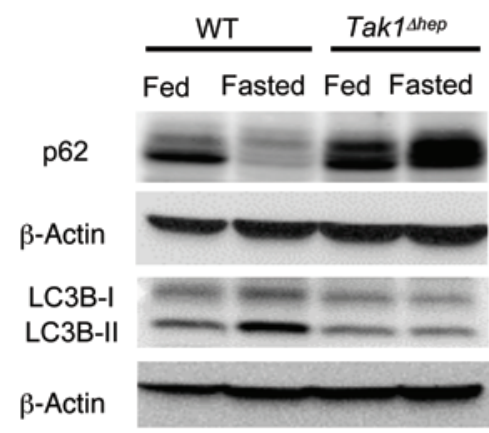

D

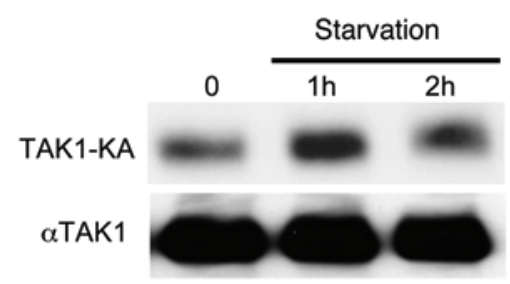

B
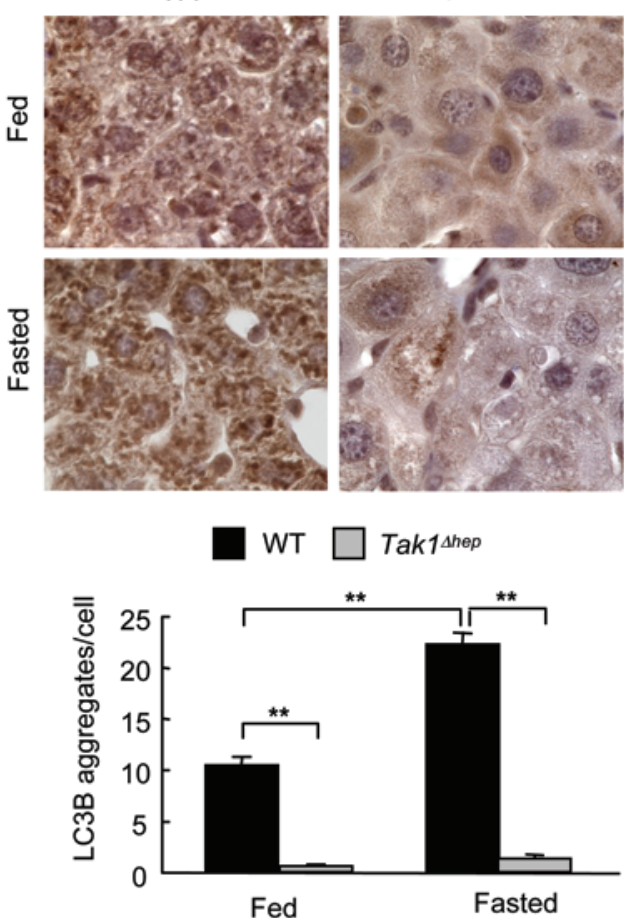

F
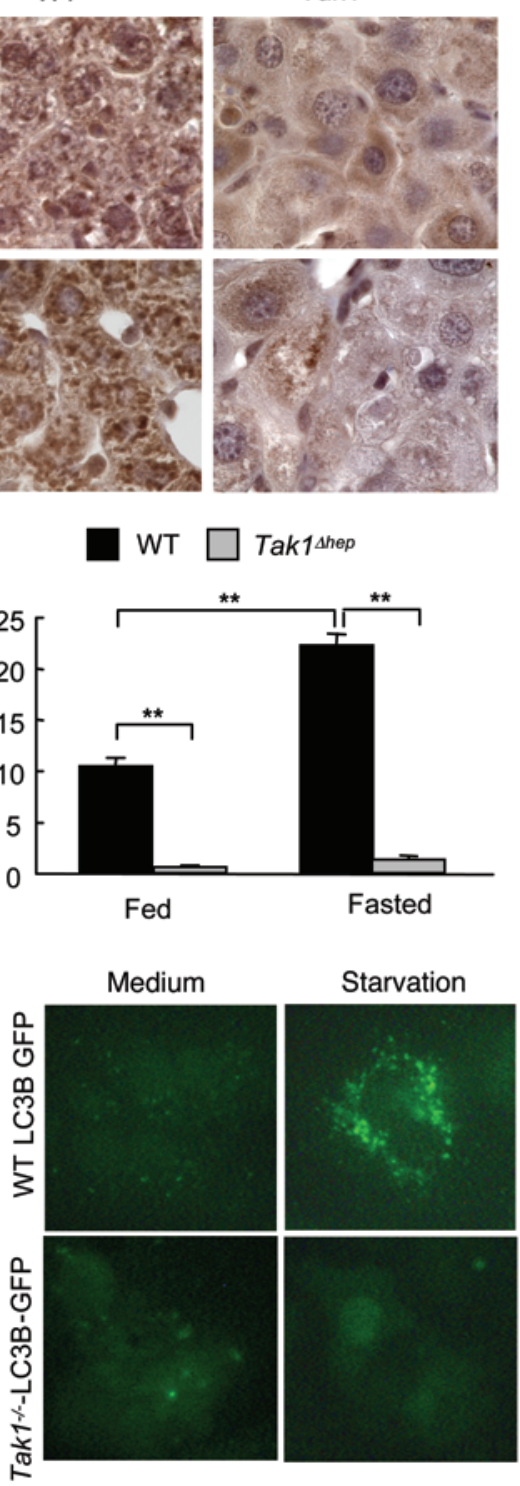

\section{E}

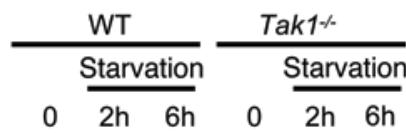

TAK1

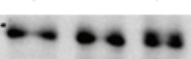

p-LKB1

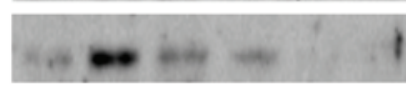

LKB1

p-AMPK

AMPK

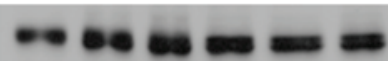

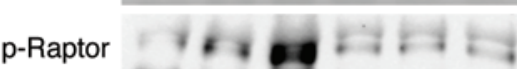

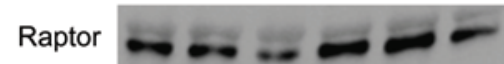

p-ULK1

ULK1

$\beta$-Actin
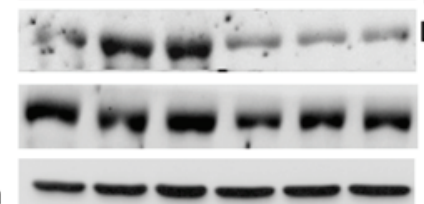

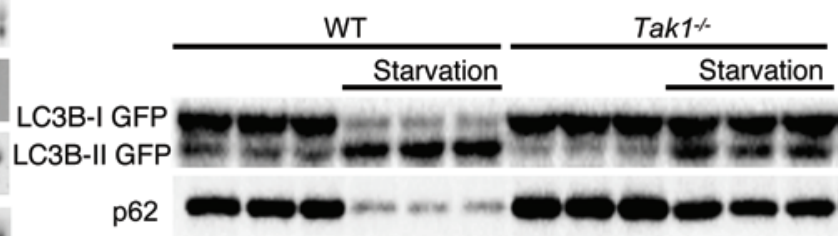

$\beta$-Actin
C

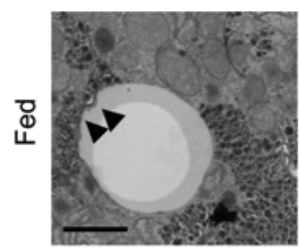

Tak1 14hep

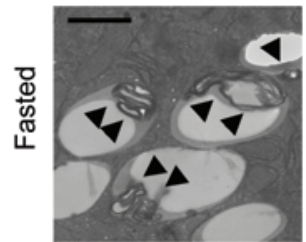

WT
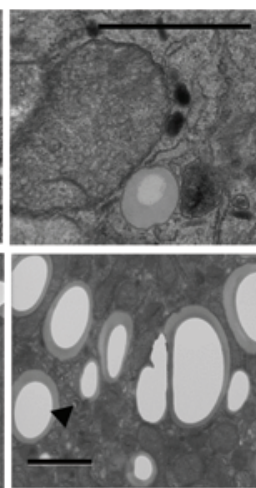

Tak1 thep
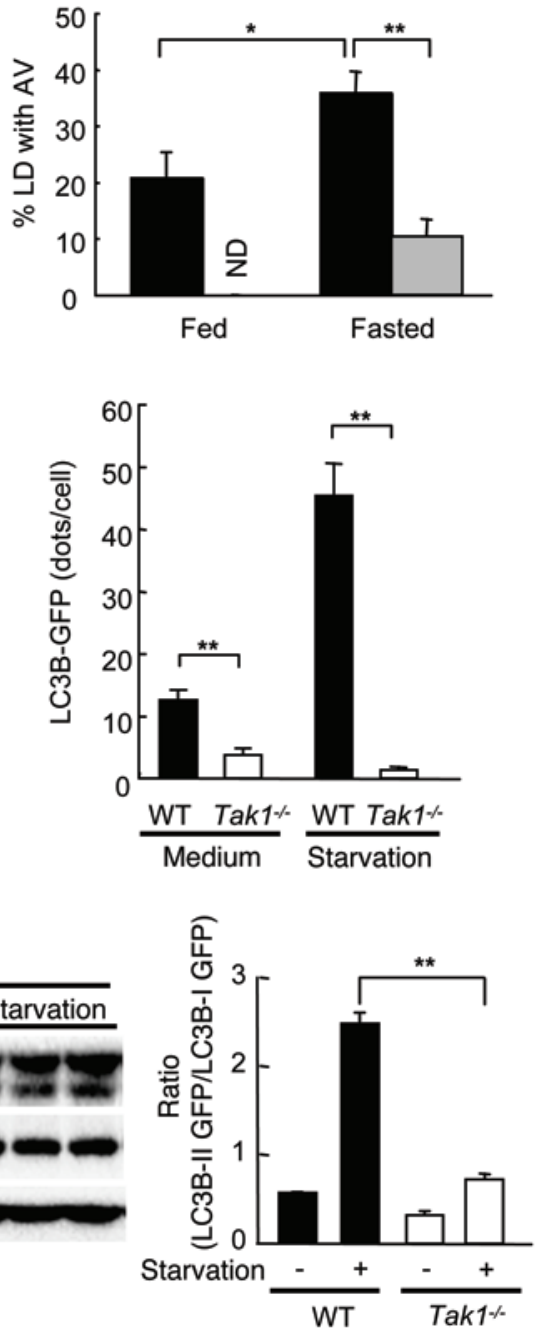

Figure 2. Defective autophagy in Tak1 $\mathbf{1}^{\text {hep }}$ mice. (A-C) One-month-old WT and Tak $1^{\text {shep }}$ mice were fasted for 12 hours ( $\mathrm{n}=5$ each). (A) Immunoblotting for hepatic expression of p62 and LC3B. (B) Immunohistochemistry for LC3B and quantification. LC3B aggregation was observed in fasted WT mice. Original magnification, $\times 1,000$. (C) EM and its quantification. Autophagosomes associated with lipid droplets are indicated with arrows. Scale bars: $1 \mu \mathrm{m}$. Lipid droplets (LD); autophasic vacuoles (AV); ND, not detected. (D) Primary hepatocytes were isolated from WT mice. TAK1 kinase activity was analyzed after 1 or 2 hours of amino acid starvation. (E) Primary hepatocytes were isolated from WT and Tak $1^{\Delta h e p}$ mice. Expressions of TAK1, p-LKB1, LKB1, p-AMPK, AMPK, p-raptor, raptor, p-ULK1, and ULK1 after 2 and 6 hours of amino acid starvation were assessed by immunoblotting. (F) Primary hepatocytes were

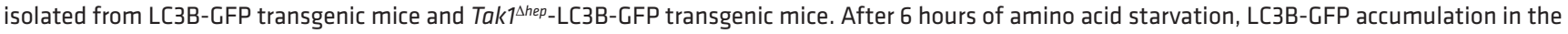
cytoplasm was assessed (top). Original magnification, $\times 200$. Black bar, WT; white bar, Tak $7^{\Delta h e p}$ mice. Immunoblotting for LC3B-GFP and p62 and quantification of the ratio of LC3B-II GFP to LC3B-I GFP (bottom). Similar results were obtained in 3 independent experiments. Data are presented as the means \pm SEM. ${ }^{*} P<0.05 ;{ }^{* *} P<0.01$. 


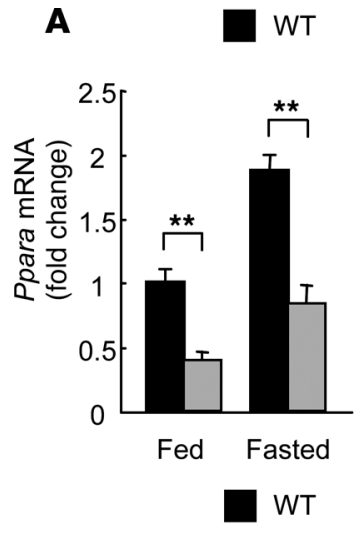

Tak1 $1^{\text {thep }}$
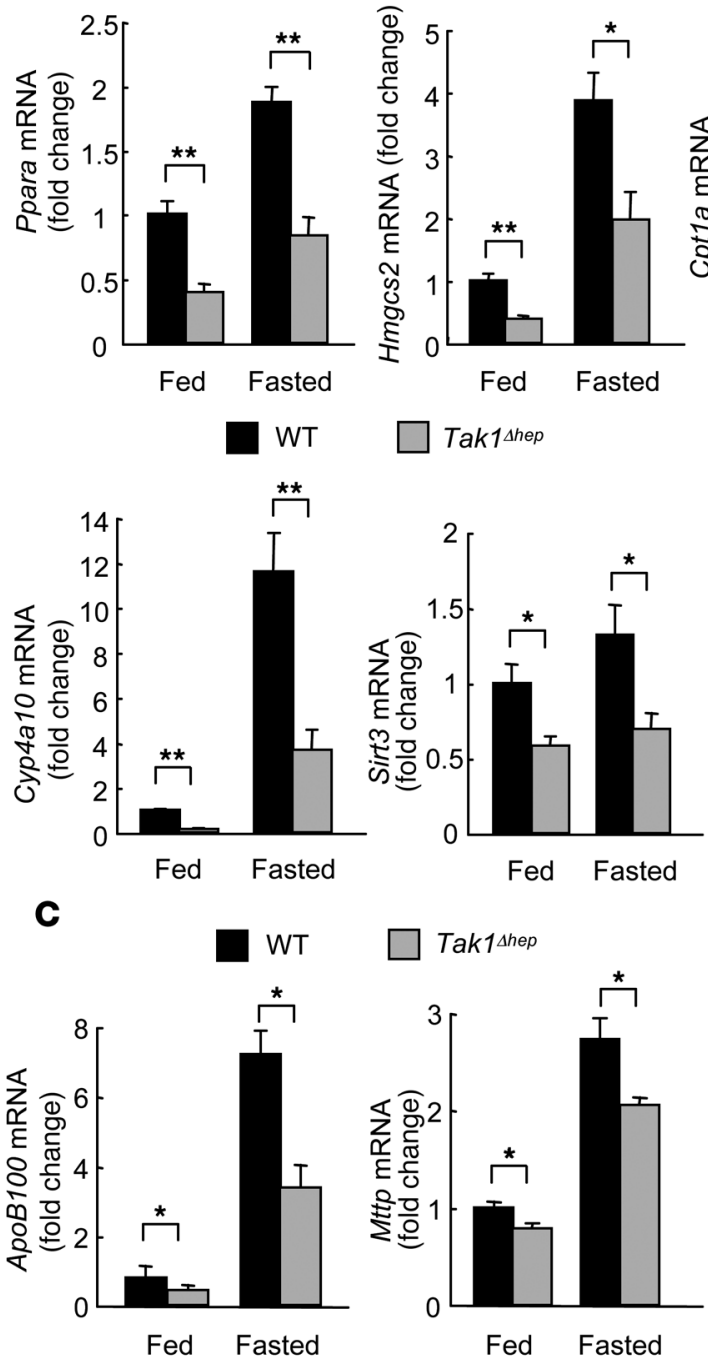
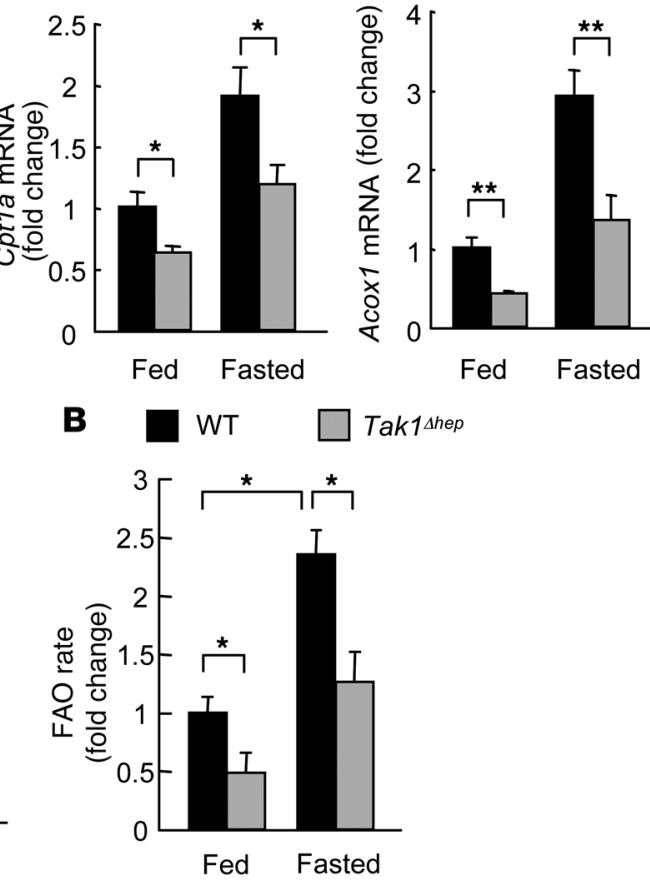

D

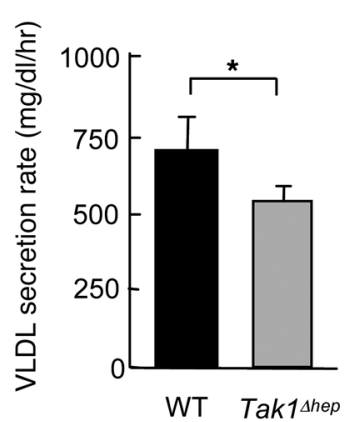

Figure 3. Gene expression involved in $\mathrm{FAO}$ and induction of FAO are suppressed in Tak $1^{\text {thep }}$ mice. One-month-old WT and Tak $7^{1 \text { hep }}$ mice were fasted for 12 hours ( $n=5$ each). (A) Hepatic expression of FAO-related genes (Ppara, Hmgcs2, Cpt1a, Acox1, Cyp4a10, and Sirt3) was measured by quantitative real-time PCR. (B) FAO was measured in WT and Tak $7^{\text {thep }}$ mice in vivo. $5 \mu \mathrm{Ci}\left[{ }^{14} \mathrm{C}\right]$ palmitate was injected into mice with or without fasting, and hepatic FAO was measured. FAO is shown as the fold change compared with WT fed controls. (C) mRNA expression of $A p o B$ and Mttp was measured by quantitative realtime PCR. (D) VLDL secretion rate was measured. Black bar, WT; gray bar, Tak $1^{\text {\hep }}$ mice. Data are presented as the means \pm SEM. ${ }^{*} P<0.05 ;{ }^{* *} P<0.01$ creased expression of FAO-related genes, such as Ppara, Hmgcs2, Cpt1a, and Sirt3 in Tak1 $1^{\text {shep }}$ mice to levels comparable to those in WT mice (Figure 5H). These findings suggest that rapamycin restored autophagy and PPAR $\alpha$ function by inhibiting excessive mTORC1 activity in Tak1 $1^{-/}$livers.

Loss of Tak1 aggravates steatohepatitis. Since advanced experimental fatty liver disease showed decreased expression of TAK1 (Supplemental Figure 7) and Tak1/- hepatocytes stored more lipid than WT hepatocytes after treatment with palmitate (Figure 4C and Supplemental Figure 6), we investigated the effect of Tak1 deficiency on the response to an HFD. Four-week-old WT and Tak $1^{\text {shep }}$ mice were placed on an HFD for 12 weeks. We observed that hepatic lipid deposition and hepatic TGs, FFAs, and cholesterol were markedly increased in Tak $1^{\text {shep }}$ mice relative to WT mice (Figure 6, A-C, and Supplemental Figure 8D). Hepatic expression of Srebp1c, Dgat1, Pparg, and Fasn was significantly upregulated in Tak1 $1^{\text {shep }}$ mice compared with that in WT mice fed an HFD (Figure 6D). Moreover, expression of inflammatory and fibrogenic genes including Tnf, Il6, Ccl2, Col1a1, and Tgfb1 and serum ALT levels were also markedly elevated in HFD-fed Tak1 $1^{\text {shep }}$ mice (Figure 6, E and F). Hepatic expression of $A f p$, an
HCC marker, was significantly increased in the HFD-fed Tak $1^{\text {shep }}$ mice compared with that observed in Tak1 $1^{\text {shep }}$ mice fed a normal chow diet (Figure 6G), suggesting that HFD feeding accelerates tumorigenesis in Tak1 ${ }^{\text {shep }}$ mice. Additionally, p62 accumulation was significantly enhanced in HFD-fed Tak1 ${ }^{\text {shep }}$ mice (Figure $6 \mathrm{H}$ and Supplemental Figure 1D), suggesting that HFD feeding further reduced autophagy in these mice. These findings collectively suggest that the impaired autophagy and increased lipogenesis caused by TAK1 deficiency render Tak $1^{\Delta h e p}$ mice highly susceptible to the adverse effects of an HFD. Interestingly, we observed no significant differences in body weight, food intake, or blood lipid and glucose levels between WT and Tak1 $1^{\text {shep }}$ mice fed an HFD (Supplemental Figure 8).

mTORC1 inhibition blocks HCC development in Tak1 ${ }^{\text {shep }}$ mice. We also examined whether excessive mTORC1 activity and defective autophagy in Tak1 $1^{\text {shep }}$ mice contribute to spontaneous hepatocarcinogenesis. Tak $1^{\text {shep }}$ mice develop spontaneous liver cancer whose histology shows a trabecular or steatotic type of HCC, with expression of typical HCC markers, including $\alpha$ fetoprotein, glypican 3, glutamine synthetase, and heat shock protein 70 (Supplemental Figure 10 and refs. 5, 8, 27, 28). Inhibition of mTORC1 

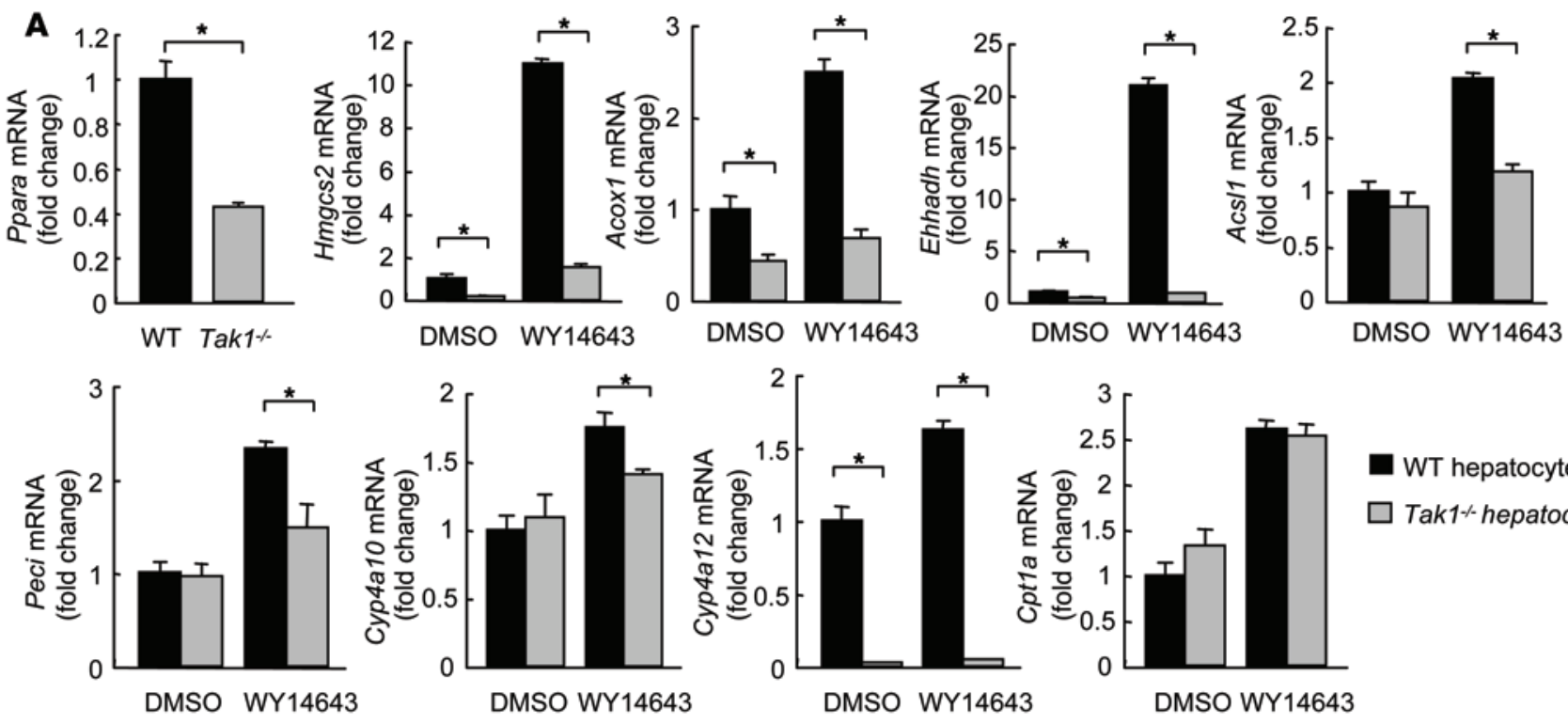

B

\section{C}
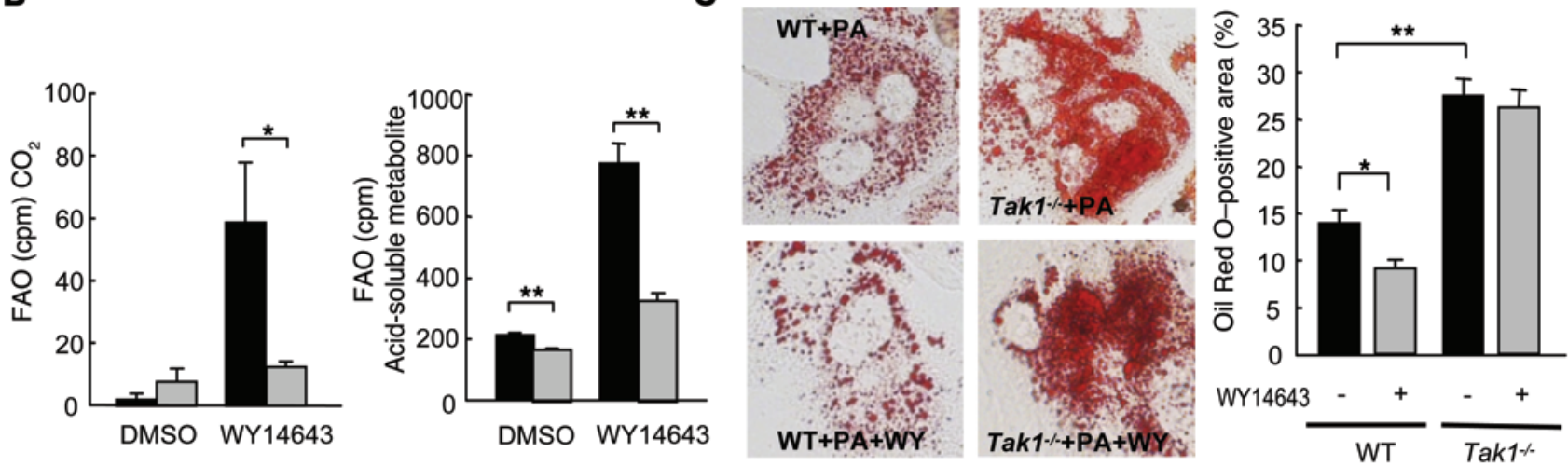

Figure 4. PPAR $\alpha$-mediated FAO is impaired in Tak $\mathbf{T}^{-/-}$hepatocytes. (A) Primary hepatocytes were isolated and incubated with palmitate (125 $\left.\mu \mathrm{M}\right)$ and PPAR $\alpha$ agonist WY14643 (30 $\mu \mathrm{M})$ for 6 hours. mRNA expression of Ppara, Hmgcs2, Acox1, Ehhadh, Acs/1, Peci, Cyp4a10, Cyp4a12, and Cpt1a was measured by quantitative real-time PCR. (B) Hepatocytes were preincubated with palmitate $(250 \mu \mathrm{M})$ and WY-14643 $(30 \mu \mathrm{M})$ for 16 hours followed by incubation with $\left[{ }^{14} \mathrm{C}\right]$-palmitate $(125 \mu \mathrm{M})$ and carnitine $(1 \mathrm{mM})$ for 2 hours. FAO was measured by scintillation counter as acid-soluble metabolites and captured $\mathrm{CO}_{2}$. Black bar, WT; gray bar, Tak1 1/- hepatocytes. (C) Hepatocytes were preincubated with palmitate $(250 \mu \mathrm{M})$ with or without WY-14643 (30 $\mu \mathrm{M})$ for 16 hours. Lipid accumulation was assessed by Oil Red 0 staining. Original magnification, $\times 200$. Similar results were obtained in 3 independent experiments. Data are presented as the means \pm SEM. ${ }^{*} P<0.05 ;{ }^{* *} P<0.01$.

with rapamycin suppressed hepatocyte death and reduced serum ALT in 1-month-old Tak1 $1^{\text {shep }}$ mice (Figure 7, A and B). Given that autophagy is strongly suppressed in HCC in Tak1 ${ }^{\text {shep }}$ mice, as demonstrated by the accumulation of $\mathrm{p} 62$ aggregates (Figure $7 \mathrm{~F}$ and Supplemental Figure 1E), we examined whether inhibition of mTORC1 activity and restoration of autophagy by rapamycin prevent HCC development. We treated Tak $1^{1 \text { hep }}$ mice with rapamycin during early (from 2 to 10 weeks after birth) or late (from 7 to 9 months of age) stages of HCC development. The early treatment significantly reduced the number of liver tumors (Figure 7C), but did not change their maximal size compared with those in the vehicle-treated Tak1 $1^{\text {hep }}$ mice (S. Inokuchi-Shimizu and E. Seki, unpublished observations). Moreover, late-stage rapamycin treatment significantly reduced the number and size of tumors and the extent of liver fibrosis in Tak $1^{\text {shep }}$ mice (Figure 7, D and E, and Supplemental Figure 9).

\section{Discussion}

Two well-established functions of TAK1 are the regulation of IKK/ NF- $\mathrm{KB}$ signaling and JNK/AP-1 signaling downstream of IL-1, TNF, TGF $\beta$, and TLRs. Through these pathways, TAK1 was suggested to modulate cell survival, proliferation, inflammation, fibrosis, and tumorigenesis, as well as lipid metabolism and insulin resistance (2). Disruption of the Ikbkg gene in hepatocytes, which encodes the IKK regulatory/scaffold subunit, combined with HFD feeding, results in exacerbated steatohepatitis, a phenotype similar to that of Tak1 ablation (29). Remarkably, TAK1 ablation in liver has a stronger tumorigenic effect than does I $k b k g$ ablation $(5,8,9)$, suggesting that enhanced susceptibility to hepatocyte death caused by the loss of IKK-NF- $\kappa$ B activity accounts for only part of the adverse consequences of Tak1 ablation. Furthermore, Ikbkg ablation in Tak1 $1^{-/}$livers attenuates HCC development $(5,8,9)$, suggesting that TAK1 suppresses tumorigenesis through an IKK-independent 

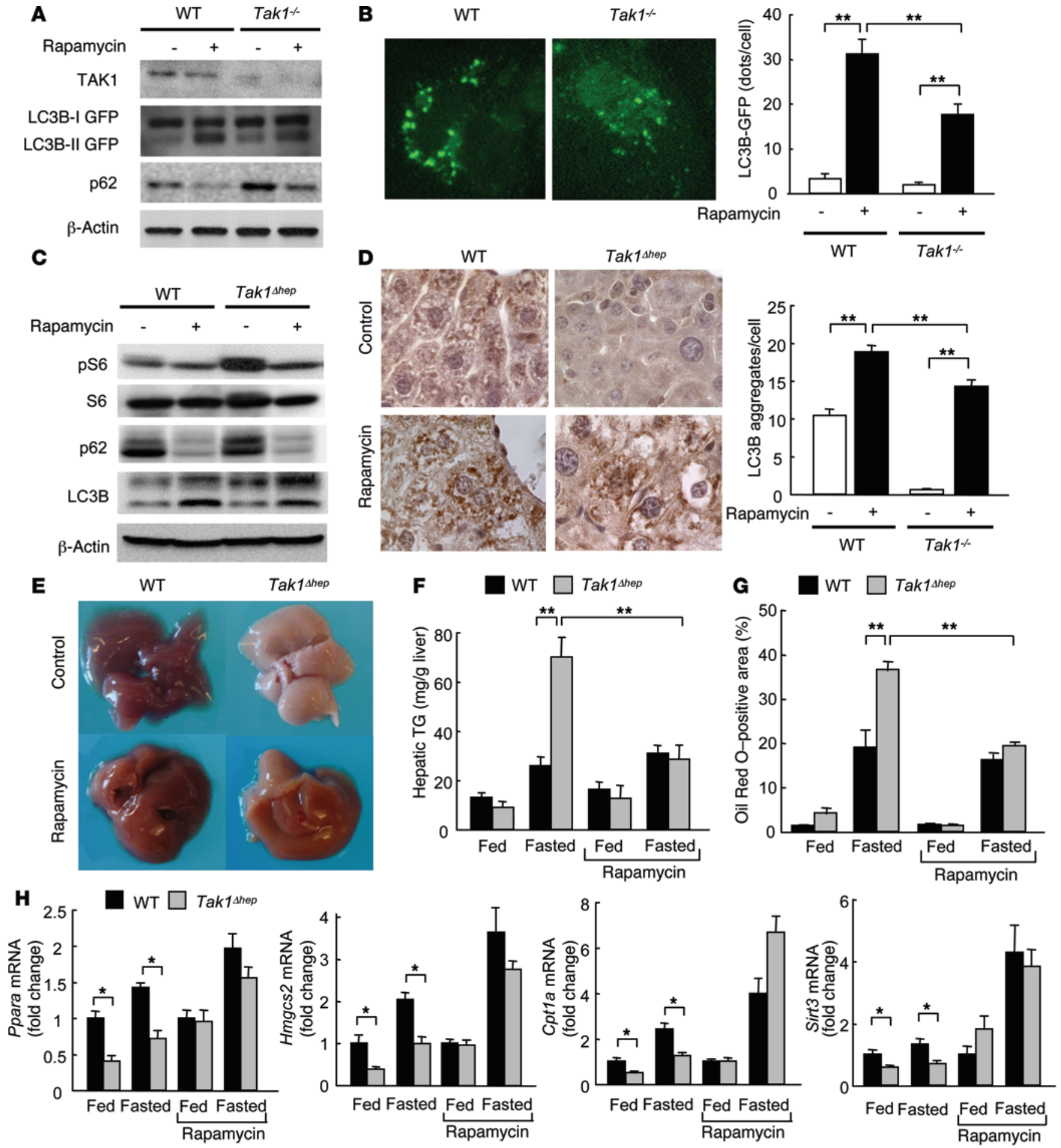

Figure 5. Rapamycin restored autophagy and FAO-related genes in Tak1/-- livers. (A and B) Primary hepatocytes were isolated from LC3B-GFP and

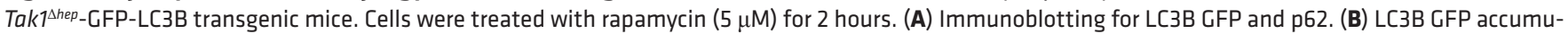
lation in cytoplasm was assessed. Original magnification, $\times 200$. White bar, vehicle; black bar, rapamycin. Similar results were obtained in 3 independent experiments. (C and D) One-month-old WT and Tak $1^{\text {hhep }}$ mice were treated with rapamycin $(5 \mathrm{mg} / \mathrm{kg}$ ) for 3 days $(n=5$ each). (C) Immunoblotting for hepatic p62 and LC3B expression. (D) Immunohistochemistry for LC3B and its quantification. LC3B aggregation in WT and Tak $7^{1 \text { hep }}$ mice treated with rapamycin. Original magnification, $\times 1,000$. (E-H) Mice treated with rapamycin $(5 \mathrm{mg} / \mathrm{kg}$ ) for 3 days followed by fasting for 12 hours ( $n=5$ each). (E) Macroscopic appearance of livers from WT and Tak $7^{\text {thep }}$ mice with fasting and rapamycin treatment. (F) Hepatic TC levels were measured, and (G) hepatic lipid deposition was assessed by quantification of Oil Red 0 staining. (H) Hepatic expression of Ppara, Hmgcs2, Cpt1a, and Sirt3 was measured by quantitative real-time PCR. Black bar, WT; gray bar, Tak1 ${ }^{\text {shep }}$ mice. Data are presented as the means \pm SEM. ${ }^{*} P<0.05 ;{ }^{* *} P<0.01$. 
A

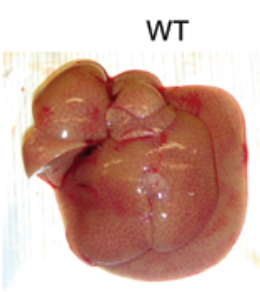

B

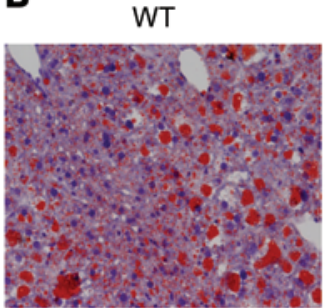

Tak1 shep

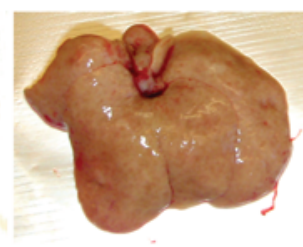

Tak14hep

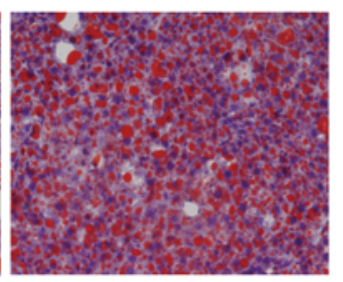

C

WT $\square$ Tak1shep

D $\square$ WT $\square$ Tak1 thep
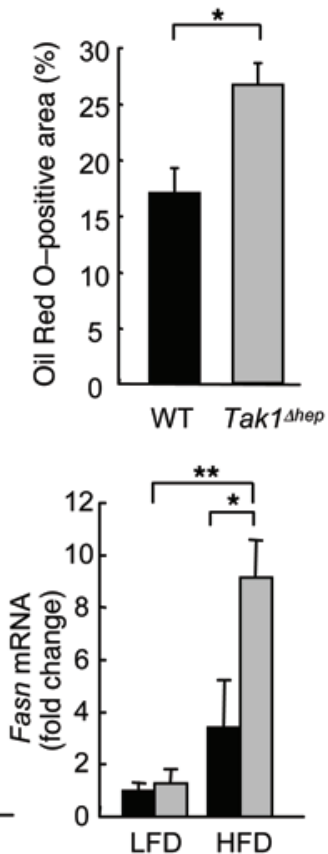

LFD HFD

E
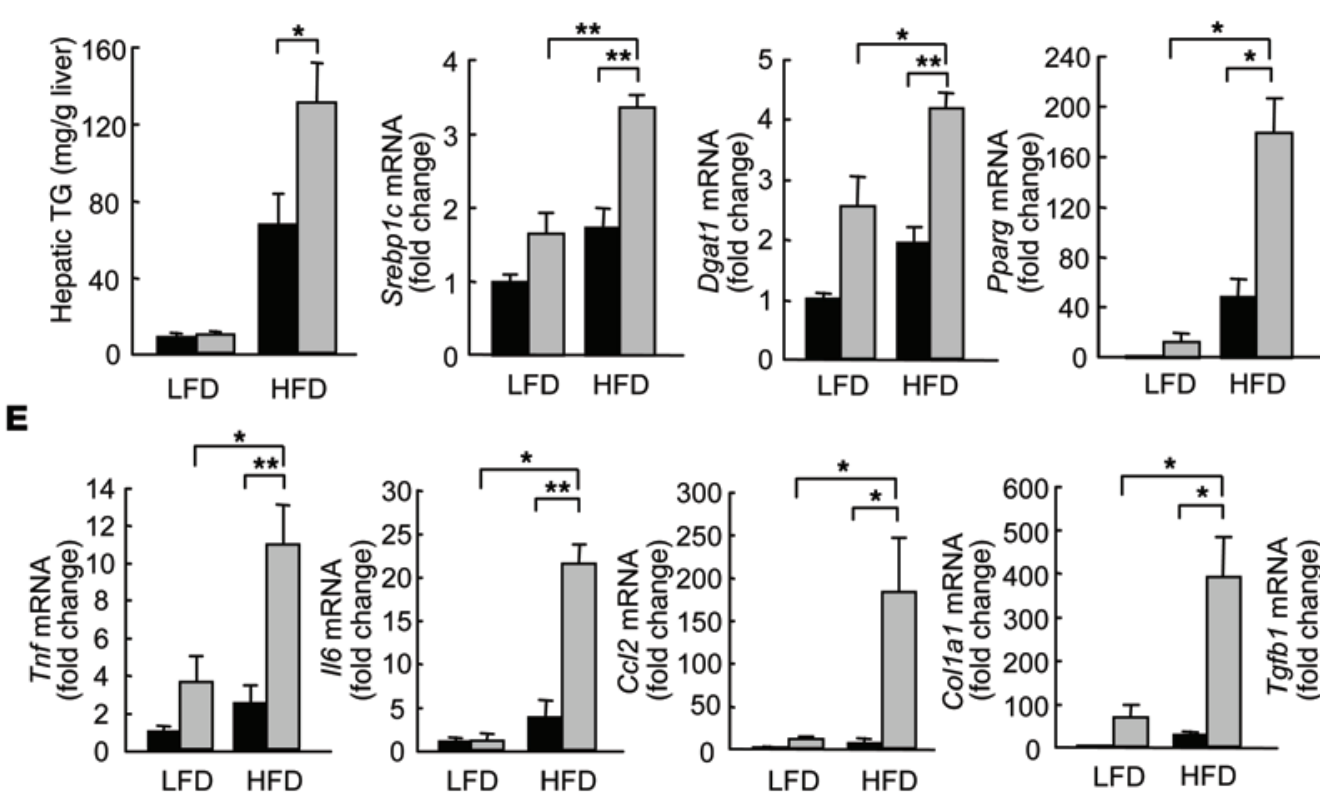

F

WT $\square$ Tak1shep
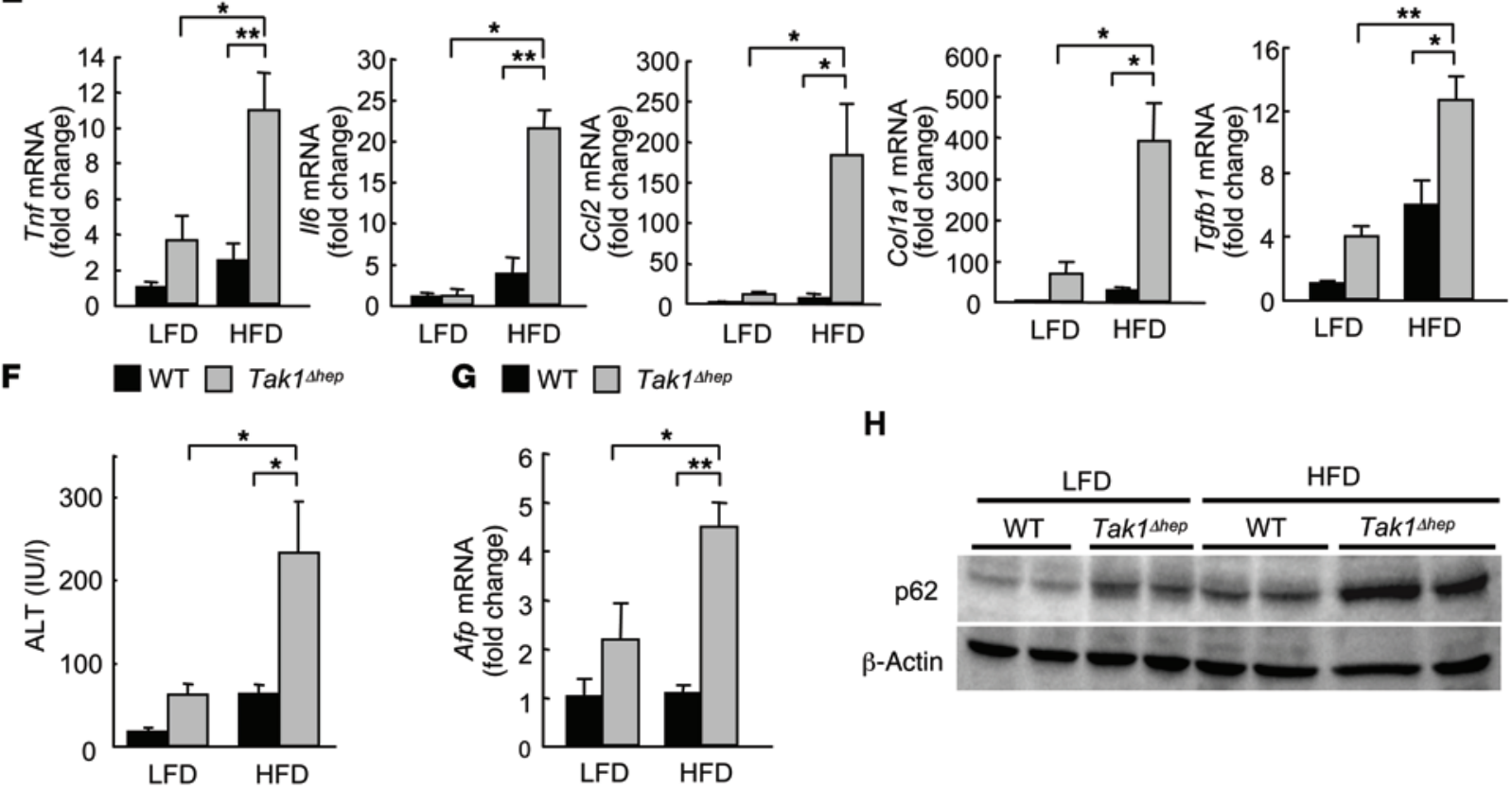

G $\square \mathrm{WT} \square T a k 1^{\Delta h e p}$

H
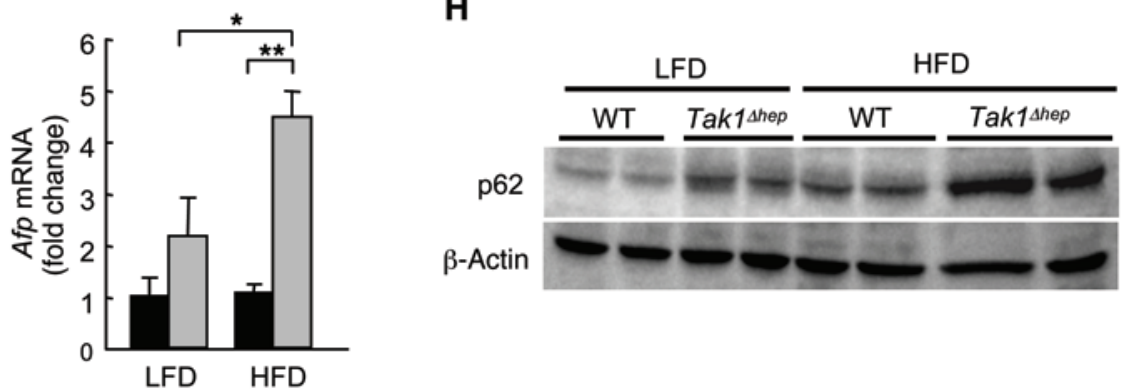

Figure 6. Loss of TAK1 enhances liver injury, lipid accumulation, inflammation, and fibrosis. One-month-old WT and Tak1 ${ }^{\text {hep }}$ mice were fed an HFD for an additional 12 weeks ( $n=5$ each). (A) Macroscopic appearance of livers. (B) Oil Red 0 staining and its quantification. Original magnification, $\times 200$. (C) Hepatic TC levels. (D) Hepatic mRNA expression of Srebp1c, Dgat1, Pparg, and Fasn was measured by quantitative real-time PCR. (E) Hepatic expression of Tnf, II6, Ccl2, Col1a1, and Tgfb1 mRNA. (F) Serum ALT levels. (G) mRNA expression of Afp. (H) Immunoblotting for p62. Black bar, WT; gray bar, Tak1 ${ }^{\text {hep }}$ mice. Data are presented as the means \pm SEM. ${ }^{*} P<0.05 ;{ }^{* *} P<0.01$.

mechanism. Since TAK1 was shown to control AMPK activity in cells $(10,30)$, we postulated that decreased AMPK activity and enhanced mTORC1 activation might contribute to some of the pathologies associated with loss of hepatic TAK1, including hepatosteatosis and HCC development. The results shown above provide strong support to this hypothesis and demonstrate that TAK1 is a physiologically relevant activator of AMPK and autophagy and a negative regulator of $\mathrm{mTORC1}$, which contribute to lipid metabolism during feeding and fasting. Furthermore, we show that exces- sive mTORC1 activity is an important contributor to the spontaneous hepatic tumorigenesis seen in Tak1 $1^{\Delta h e p}$ mice. In this respect, the liver phenotype in Tak $1^{\Delta h e p}$ mice resembles that of $T s c 1^{\Delta h e p}$ mice, whose hepatocytes lack TSC1, a negative regulator of mTORC1 (31). In both strains of mice, rapamycin inhibits HCC development and hepatic steatosis $(14,31)$

Our data demonstrate that starvation-induced LKB1 and AMPK phosphorylation requires TAK1 in hepatocytes, although the mechanism by which TAK1 controls LKB1 and AMPK activ- 
A

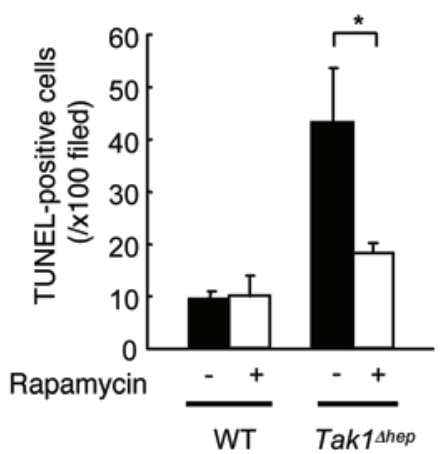

B

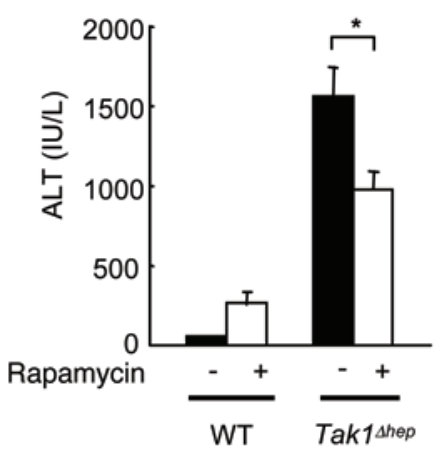

C Rapamycin treatment during early stage

D Rapamycin treatment during late stage

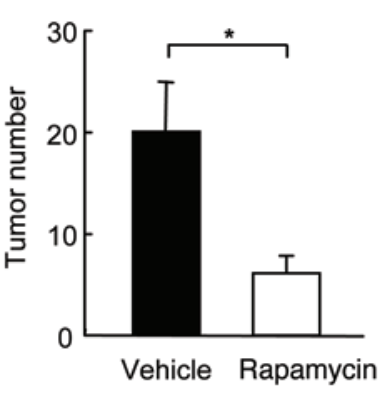

Tak1 thep

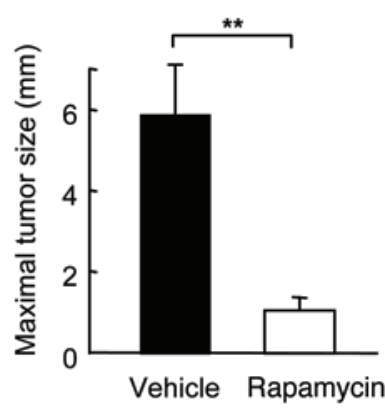

Tak1 thep

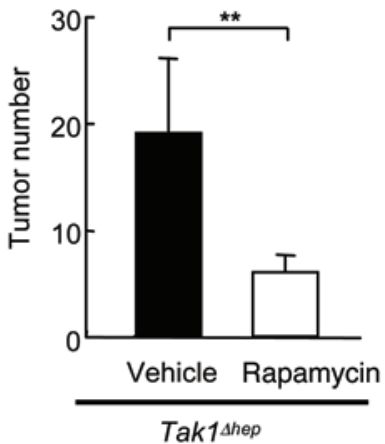

E

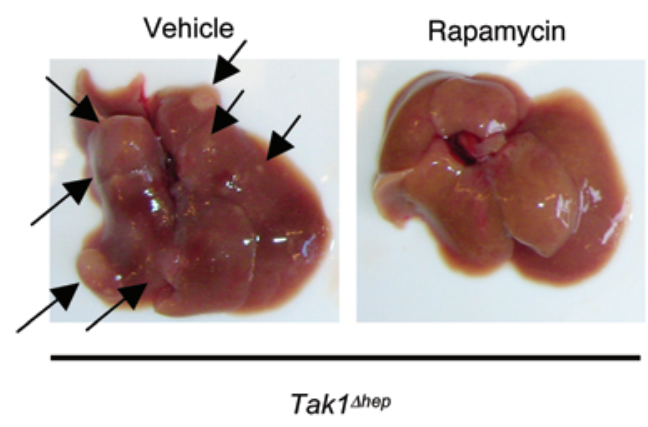

Tak1 thep

$\mathbf{F}$

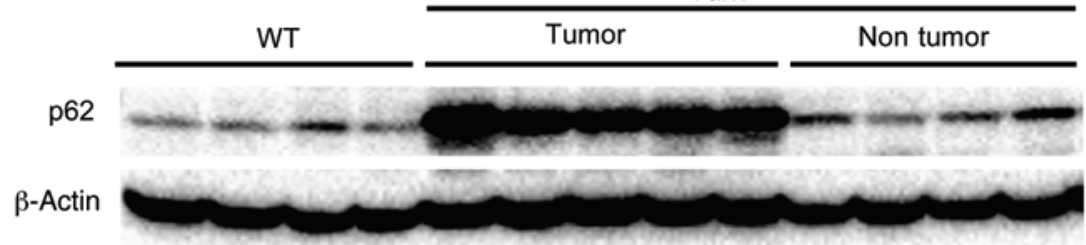

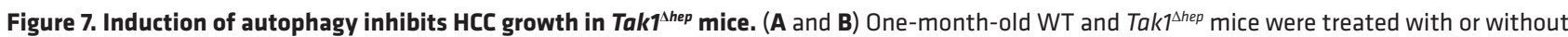
rapamycin $(5 \mathrm{mg} / \mathrm{kg})$ for 3 days $\left(n=5\right.$ each). (A) Hepatocyte apoptosis was assessed by TUNEL staining. (B) Serum ALT levels. (C) Tak ${ }^{1 \text { hep }}$ mice were treated with rapamycin $(5 \mathrm{mg} / \mathrm{kg}$ ) twice weekly from 2 to 10 weeks of age, and the livers were harvested from the mice at 36 weeks of age ( $n=12$ each). Macroscopically visible tumors were counted. (D and E) Tak $1^{\text {thep }}$ mice were treated with rapamycin $(5 \mathrm{mg} / \mathrm{kg}$ ) twice weekly from 28 to 36 weeks of age, and then the livers were harvested $(n=12)$. (D) Macroscopically visible tumors were counted, and maximal size was measured. (E) Macroscopic appearance of the liver. (F) Expression of p62 in HCC in Tak1 $1^{\text {hhep }}$ mice as assessed by immunoblotting for p62. (A-D) Black bar, vehicle; white bar, rapamycin. Data are presented as the means \pm SEM. ${ }^{*} P<0.05 ;{ }^{*} P<0.01$.

ities remains elusive $(10,30)$. Activated AMPK then phosphorylates raptor, which inhibits mTORC1 activity, resulting in stimulation of autophagy (Supplemental Figure 11 and refs. 12, 13). The contribution of TAK1 to starvation-induced autophagy, however, has been controversial. One study reported that TAK1 is required for starvation-induced autophagy $(10,19)$, while another study showed that TAK1 is dispensable for starvation-induced autophagy but is required for TRAIL-induced autophagy $(10,19)$. Given the different cell types examined in the previous studies and in our current study, it is conceivable that the requirement of TAK1 in starvation-induced autophagy is largely cell-type specific and may reflect differential effects on AMPK. Although our results show that starvation- and metformin-induced AMPK activation and autophagy are dependent on TAK1 and that ectopic expression of activated AMPK reverses the autophagy defect in $\mathrm{Tak1}^{-/ \text {- }}$ hepatoc- ytes, suggesting TAK1-mediated autophagy through AMPK. However, a recent report demonstrated that TAK1 also participates in autophagy through the inhibition of p70S6K phosphorylation by interacting with raptor independently of AMPK (32). This suggests that the increased basal activity of mTORC1 and suppressed basal autophagy in $\mathrm{Tak1}^{-1-}$ hepatocytes may be mediated by a direct effect of TAK1 on mTORC1-p70S6K activity. It should be noted that TAK1-binding protein 2 (TAB2) and TAB3 also regulate autophagy through their effects on beclin 1 and may therefore be involved in TAK1-regulated autophagy in the liver $(33,34)$. In addition, TAK1 deficiency results in reduced expression of several autophagy genes, including $A \operatorname{tg} 3, \operatorname{Atg} 5, A \operatorname{tg} 8$, and Ulk1, providing another mechanism by which TAK1 can influence autophagy.

In addition to autophagy, mTORC1 inhibits FAO by suppressing PPAR $\alpha$ expression and function (14). PPAR $\alpha$ is a pow- 
erful FAO inducer involved in lipid breakdown and energy production through induction of FAO-related genes, including Hmgcs2, Acox1, and Cpt1a. Absence of Tsc1 causes a strong mTORC1 activation that decreases expression of PPAR $\alpha$ and its target genes, thereby demonstrating another similarity between $T a k 1^{\Delta h e p}$ and $T s c 1^{\Delta h e p}$ mice (14). Restoration of PPAR $\alpha$ activity by ectopic PPAR $\alpha$ introduction or treatment with a PPAR $\alpha$ agonist, however, does not restore PPAR $\alpha$ function in $T s c 1^{\Delta \text { hep }}$ mice, suggesting that strong mTORC1 overactivation may also affect functions of cofactors (e.g., NCoR1) that require PPAR $\alpha$-mediated functions (14). Due to overactivation of mTORC1 in Tak1/-- livers, expression of PPAR $\alpha$ and its target genes, as well as FAO, are suppressed, which may contribute to elevated hepatic steatosis in Tak1 $1^{\text {shep }}$ mice upon fasting or after HFD feeding. Moreover, TAK1 deficiency inhibited expression of SIRT3, which is required for deacetylation and activation of LCAD and HMGCS2 $(17,35)$, suggesting that FAO may also be suppressed through inhibition of SIRT3 in Tak1 $1^{-/-}$hepatocytes.

We found that inhibition of mTORC1 with rapamycin successfully restored autophagy and PPAR $\alpha$ function and thereby reduced lipid accumulation in the $\mathrm{Tak1}^{-/-}$livers. This beneficial effect of mTORC1 inhibition was largely dependent on restoration of autophagy, further suggesting that the autophagic defect is responsible for the increase in hepatic steatosis caused by the TAK1 deficiency. As previously observed in Tsc1 $1^{\Delta h e p}$ mice (31), we found that rapamycin treatment also blocked fibrosis and HCC development in Tak1 $1^{\text {shep }}$ mice.

Hypernutrition causes an mTORC1 activation that contributes to lipogenesis through activation of SREBP-1c and PPAR $\gamma$ and inhibition of lipid breakdown through suppression of autophagy $(12,18)$. Furthermore, hepatic TAK1 expression is decreased during dietary and genetic obesity (Supplemental Figure 7), allowing for an additional enhancement of mTORC1 activity that increases lipogenesis and suppresses autophagy and FAO. Degradation of p62-bound polyubiquitinated proteins within autophagosomes is mediated by autophagy (22). The decrease in autophagy in $\mathrm{Tak1}^{-/-}$hepatocytes results in accumulation of p62, which leads to further activation of mTORC1, as recently described $(36,37)$. The decrease in autophagy and the increase in mTORC1 activity resulted in enhanced development of hepatosteatosis in HFD-fed Tak1 $1^{\Delta h e p}$ mice. In addition, we found that liver inflammation and fibrosis were substantially increased in Tak1 $1^{\text {shep }}$ mice after HFD feeding. Despite extensive investigations, the trigger(s) or factor(s) that cause progression from simple steatosis to steatohepatitis remain unknown. Given the fact that hepatic TAK1 expression is suppressed in advanced fatty liver disease, factors that lead to a further decrease in TAK1 activity or increase mTORC1 activation may contribute to the progression from simple steatosis to steatohepatitis.

Since the IKK/NF- $\kappa B$ pathway is known to prevent HCC formation and TAK1 acts upstream of the IKK/NF- $\mathrm{BB}$ pathway, it was suggested that spontaneous HCC development in Tak1 $1^{\text {shep }}$ mice is

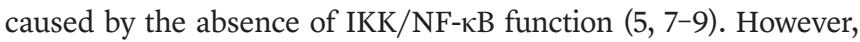
the present results indicate that excessive mTORC1 activity is equally important or even more important for spontaneous HCC development in Tak1 $1^{\text {shep }}$ mice. It has been reported that the complete absence of autophagy causes the development of benign liver tumors with $\mathrm{p} 62$ accumulation rather than the development of HCC $(38,39)$. As recently shown in the pancreas $(40)$, malignant progression seems to require a certain level of autophagy, thus explaining why Tak1 $1^{\text {shep }}$ mice, in which autophagy is reduced but not completely absent, develop malignant HCC, whereas Alb-Cre Atg5 ${ }^{f / f l}$ or $A l b$-Cre Atg $7^{f / f}$ mice only develop benign adenomas.

Despite aggravated hepatosteatosis, body weight and insulin resistance were not altered in HFD-fed Tak $1^{\text {shep }}$ mice compared with WT mice fed an HFD, which is similar to the phenotype of Ikbkg ${ }^{\Delta h e p}$ mice (29). This indicates a disassociation between hepatosteatosis and insulin resistance. Hepatic TAK1 and IKK-NF- $\kappa$ B prevent hepatosteatosis, but do not affect systemic insulin sensitivity.

Remarkably, in $30 \%$ of human prostate cancers, chromosome 6q15.1 containing the Tak1 locus is deleted (41). Although clinical evidence for TAK1 deficiency in human HCC is still lacking, deficiency in TAK1 may promote tumor development due to overactivation of mTORC1 and suppression of autophagy. Since rapamycin treatment of HCC patients after liver transplantation results in reduced HCC recurrence (42), it is plausible that inhibition of mTORC1 activity may become a useful therapy for HCC (43). In summary, TAK1 is a physiological regulator of AMPK and autophagy and a negative regulator of mTORC1. TAK1 deficiency causes excessive mTORC1 activation and autophagy defects, which contribute to enhancement of hepatosteatosis and spontaneous hepatic tumorigenesis.

\section{Methods}

Mice, diets, and treatments. Albumin-Cre recombinase transgenic mice were purchased from The Jackson Laboratory. Mice carrying the floxed allele of Tak1 (Tak1 $1^{A / f}$ mice) have been described previously (44). These mouse lines were crossed to generate $\mathrm{Alb}-\mathrm{Cr} \mathrm{f}^{/+} \mathrm{Tak} 1^{\mathrm{Alf} /}$ mice ( $\mathrm{Tak} 1^{\text {shep }}$ ) on a C57BL6 background. Cre-negative animals were used as WT controls. LC3B-GFP mice were provided by N. Mizushima (Tokyo Medical and Dental University, Tokyo, Japan) (24). Mice were given free access to an autoclaved regular chow diet (LFD) or an HFD (composed of $59 \%$ fat, $15 \%$ protein, and $26 \%$ carbohydrates based on caloric content; BioServ) and water. In some experiments, mice underwent 12-hour fasting to induce autophagy and/or were subjected to rapamycin treatment. For short rapamycin treatment, $5 \mathrm{mg} / \mathrm{kg}$ /day of rapamycin or vehicle was administered to mice for 3 days. For tumor studies, $5 \mathrm{mg} / \mathrm{kg}$ of rapamycin or vehicle were given to Tak $1^{\text {shep }}$ twice a week for 2 months. Mice were also treated with CQ $(60 \mathrm{mg} / \mathrm{kg}) 24$ hours prior to sacrifice.

Mouse tissue processing and histological examination. Mouse tissues for histological examination were fixed in $10 \%$ or $4 \%$ neutral buffered formalin phosphate (Fisher Scientific) and were subsequently embedded in paraffin or OCT compound, respectively. They were sliced into 5- $\mu \mathrm{m}$ sections for Oil Red O staining, TUNEL staining, or Sirius red staining, and for immunohistochemistry. For immunostaining, liver sections were incubated with anti-LC3B Ab (MBL International) or anti-p62 Ab (Progen). LC3B aggregates were evaluated and quantified by 2 independent pathologists (Y.S. Roh and K. Asahina). TUNEL-positive cells and Sirius red-positive areas were counted and measured, respectively, for 10 low-powered $(\times 100)$ fields per slide and quantified using Image J software (NIH).

Western blot analysis. Protein extracts were electrophoresed, blotted, and then incubated with antibodies against TAK1, phosphorylated ribosomal S6, ribosomal S6, LC3B, phosphorylated LKB1 (p-LKB1), LKB1, p-AMPK, AMPK, p-raptor, raptor, p-ULK1, 
ULK1 (Cell Signaling Technology), $\beta$-actin (Sigma-Aldrich), GFP (Abcam), and p62/SQSTM1 (Progen), with appropriate secondary HRP-conjugated antibodies, and then developed.

Measurement of TAK1 kinase activity. An in vitro kinase assay was performed to measure TAK1 kinase activity as described previously (45). Briefly, cell pellets were lysed in lysis buffer (20 mM HEPES [pH 7.6], $250 \mathrm{mM} \mathrm{NaCl}, 0.5 \% \mathrm{NP}-40,20 \mathrm{mM} \beta$-glycerophosphate, $1 \mathrm{mM}$ EDTA, $20 \mathrm{mM}$ p-nitrophenyl phosphate, $0.1 \mathrm{mM} \mathrm{Na}_{3} \mathrm{VO}_{4}$, $1 \mathrm{mM}$ DTT, and $1 \mathrm{mM}$ PMSF). An anti-TAK1 antibody (Cell Signaling Technology) was used to immunoprecipitate the TAK1 protein complex, which was then incubated with MKK6 substrate (Millipore) in the presence of $\left[\gamma^{-32} \mathrm{P}\right]$ ATP for 30 minutes at $30^{\circ} \mathrm{C}$. The samples were boiled in SDS loading buffer, and the proteins were separated by SDS-PAGE. Proteins were then transferred to PVDF membranes and exposed to autoradiography. The membranes were subjected to Western blotting with anti-TAK1 antibody.

Quantitative real-time PCR analysis. RNA extracted from liver was subjected to reverse transcription and subsequent PCR using a CFX96 real-time PCR system (Bio-Rad). PCR primer sequences are listed in Supplemental Table 5. Expression of the respective genes was normalized to $18 \mathrm{~S}$ rRNA as an internal control.

Assessment of in vivo FAO. To measure hepatic FAO in vivo, $5 \mu \mathrm{Ci}\left[{ }^{14} \mathrm{C}\right]$ palmitate was injected into WT and Tak1 $1^{\text {shep }}$ mice, and livers were harvested 2 minutes following the injection (46). FAO acid-soluble metabolites and captured $\mathrm{CO}_{2}$ were measured using a scintillation counter.

Measurement of VLDL TG secretion rates. Mice were fasted for 4 hours and were intraperitoneally injected with $400 \mu \mathrm{l} \mathrm{P}-407$ (1 $\mathrm{mg} / \mathrm{g}$ ) (Calbiochem) solution in PBS (47). Blood was collected from the tail vein prior to injection ( 0 hour) and at 1, 2, 4, and 8 hours after injection. TGs were measured, and the VLDL TG secretion rate was calculated as the slope of the plasma TG concentration versus the time after linear regression and expressed in $\mathrm{mg} / \mathrm{dl} /$ hour.

Hepatocyte isolation and treatments. Hepatocytes were isolated from WT, Tak1 $1^{\text {shep }}$, LC3B-GFP, and LC3B-GFP-Tak1 $1^{\text {shep }}$ mice. After cell attachment, hepatocytes were serum starved for 8 hours and then stimulated by amino acid starvation using Krebs Ringer solution, metformin $(2 \mathrm{mM})$, or rapamycin $(5 \mu \mathrm{M})$ (Sigma-Aldrich) for the indicated time periods. In some experiments, hepatocytes were treated with PPAR $\alpha$ agonist WY-14643 $(30 \mu \mathrm{M})$ (Sigma-Aldrich) in combination with $125 \mu \mathrm{M}$ palmitate for 6 hours. PPAR $\alpha$ and PPAR $\gamma$ target genes were measured by quantitative PCR (16). Control siRNA or siRNA for Ppara or Atg5 (Thermo Scientific) for silencing the indicated genes and adenovirus encoding constitutive active AMPK or $L a c Z$ as a control have been used in some experiments (48). To examine FAO in hepatocytes in vitro, cells were preincubated with $250 \mu \mathrm{M}$ palmitate and $30 \mu \mathrm{M} \mathrm{WY}-14643$ for 16 hours followed by incubation with $125 \mu \mathrm{M}\left[{ }^{14} \mathrm{C}\right]$-palmitate and $1 \mathrm{mM}$ carnitine for 2 hours. FAO acid-soluble metabolites and captured ${ }^{14} \mathrm{CO}_{2}$ were measured by scintillation counter (16).

Glucose tolerance tests. For the glucose tolerance test, baseline glucose levels were measured from mice fasted for 6 hours ( 0 minutes). Then, $1 \mathrm{~g} / \mathrm{kg}$ glucose was administered via intraperitoneal injection, and glucose levels were measured 20, 40, 60, and 120 minutes following glucose loading.

Transmission electron microscopy. Mouse liver was fixed through whole-body perfusion with Ringer's solution and fixation buffer composed of $2 \%$ PFA and $2.5 \%$ glutaraldehyde (Ted Pella Inc.) plus $3 \mathrm{mM}$ calcium chloride in $0.1 \mathrm{M}$ sodium cacodylate buffer $\mathrm{pH} 7.4$ ) (49). Dissected pieces of mouse liver were placed in fixation buffer on ice for 2 hours. The samples were washed 5 times with buffer consisting of $0.1 \mathrm{M}$ sodium cacodylate plus $3 \mathrm{mM}$ calcium chloride ( $\mathrm{pH}$ 7.4) on ice. The liver pieces were vibratomed with a Leica VT 1000 s vibratome at $80 \mu \mathrm{m}$, then the tissues were postfixed with $1 \%$ osmium tetroxide, $0.8 \%$ potassium ferrocyanide, and $3 \mathrm{mM}$ calcium chloride in $0.1 \mathrm{M}$ sodium cacodylate ( $\mathrm{pH}$ 7.4) for 1 hour, washed 3 times with ice-cold distilled water, en bloc stained with $2 \%$ uranyl acetate at $4^{\circ} \mathrm{C}$ for overnight, dehydrated through graded ethanol solutions, and embedded in Durcupan ACM resin (Fluka, SigmaAldrich). Ultrathin 80-nm sections were made with a Leica Ultracut UCT ultramicrotome, and sections were poststained with uranyl acetate and lead salts prior to imaging using a JEOL 1200 EX transmission EM operated at $80 \mathrm{kV}(49)$.

Microarray gene expression profiling. Total RNA was extracted from frozen liver tissues ( $n=2$ for each strain) using the RNeasy kit (QIAGEN). Biotinylated cRNA was prepared using the Illumina RNA Amplification Kit (Ambion, Life Technologies). cRNA was hybridized to MouseRef-8 v2.0 Expression BeadChip Arrays (Illumina) and scanned on the Illumina BeadArray reader. Data analysis was carried out using Illumina BeadStudio software. We applied our variance modeled posterior inference with regional exponentials (VAMPIRE) microarray analysis web site to the gene expression data to identify statistically significant differences between gene expression profiles from the subject groups (50-52). We identified significantly enriched gene ontology and KEGG pathway annotation terms in expression datasets using GOby, which is part of the VAMPIRE microarray suite (52). We corrected for errors associated with multiple comparisons (accounting for the number of genes examined) using the stringent Bonferroni correction at a $5 \%$ significance level cutoff $\left(\alpha\right.$ Bonf $\left.=0.05, \alpha=9.72876211230883 \times 10^{-7}\right)$. Microarray data were deposited in the NCBI's Gene Expression Omnibus database (GEO GSE51962).

Statistics. Data are presented as the means \pm SEM. Differences between 2 groups were compared using a 2-tailed, unpaired Student's $t$ test. $P$ values less than 0.05 were considered significant.

Study approval. All mice received humane care according to the NIH recommendations outlined in their Guide for the Care and Use of Laboratory Animals (NIH publication no. 85-23. Revised 1985. All animal experiments were approved by the IACUC of UCSD.

\section{Acknowledgments}

LC3B-GFP transgenic mice were provided by Noboru Mizushima (Tokyo Medical and Dental University, Tokyo, Japan). Pingping Li (UCSD, Department of Medicine) provided liver samples from $o b / o b$ mice. This study was supported by NIH grants R01AA02172 (to E. Seki), R01DK085252 (to E. Seki), R01CA155120 (to M. Karin), P42 ES010337 (to E. Seki, D.A. Brenner, M. Karin, and M.H. Ellisman), and R01AA020753 (to K. Asahina); by a pilot grant from the UCSD Digestive Diseases Research Development Center (DK080506, to E. Seki); by the National Natural Science Foundation of China (30500658 and 81370550, to L. Yang); a Postdoctoral Fellowship (to Y.S. Roh) and a Liver Scholar Award (to E.J. Park) from the American Liver Foundation; a postdoctoral fellowship from Susan G. Komen for the Cure (KG111506, to X. 
Wu); and a Postdoctoral Fellowship for Research Abroad, a Research Fellowship for Young Scientists from the Japan Society for the Promotion of Science, and a Uehara Memorial Foundation Fellowship (to K. Taniguchi).
Address correspondence to: Ekihiro Seki, UCSD, 9500 Gilman Drive, MC\# 0063, Biomedical Research Facility 2, Rm 4A21, La Jolla, California 92093-0063, USA. Phone: 858.822.3347; E-mail: ekseki@ucsd.edu.
1. Shim JH, et al. TAK1, but not TAB1 or TAB2, plays an essential role in multiple signaling pathways in vivo. Genes Dev. 2005;19(22):2668-2681.

2. Sakurai H. Targeting of TAK1 in inflammatory disorders and cancer. Trends Pharmacol Sci. 2012;33(10):522-530.

3. Seki E, Brenner DA, Karin M. A liver full of JNK: signaling in regulation of cell function and disease pathogenesis, and clinical approaches. Gastroenterology. 2012;143(2):307-320.

4. Karin M. Nuclear factor-kappaB in cancer development and progression. Nature. 2006;441(7092):431-436.

5. Inokuchi S, et al. Disruption of TAK1 in hepatocytes causes hepatic injury, inflammation, fibrosis, and carcinogenesis. Proc Natl Acad Sci U S A. 2010;107(2):844-849.

6. Yang L, et al. Transforming growth factor-beta signaling in hepatocytes promotes hepatic fibrosis and carcinogenesis in mice with hepatocytespecific deletion of TAK1. Gastroenterology. 2013;144(5):1042-1054 e1044.

7. Maeda S, Kamata H, Luo JL, Leffert H, Karin M. IKKbeta couples hepatocyte death to cytokine-driven compensatory proliferation that promotes chemical hepatocarcinogenesis. Cell. 2005;121(7):977-990.

8. Bettermann K, et al. TAK1 suppresses a NEMOdependent but NF- $\kappa \mathrm{B}$-independent pathway to liver cancer. Cancer Cell. 2010;17(5):481-496.

9. Luedde T, et al. Deletion of NEMO/IKK $\gamma$ in liver parenchymal cells causes steatohepatitis and hepatocellular carcinoma. Cancer Cell. 2007;11(2):119-132.

10. Herrero-Martin G, et al. TAK1 activates AMPK-dependent cytoprotective autophagy in TRAIL-treated epithelial cells. EMBO J. 2009;28(6):677-685.

11. Russo GL, Russo M, Ungaro P. AMP-activated protein kinase: A target for old drugs against diabetes and cancer. Biochem Pharmacol. 2013;86(3):339-350.

12. Zoncu R, Efeyan A, Sabatini DM. mTOR: from growth signal integration to cancer, diabetes and ageing. Nat Rev Mol Cell Biol. 2011;12(1):21-35.

13. Egan DF, et al. Phosphorylation of ULK1 (hATG1) by AMP-activated protein kinase connects energy sensing to mitophagy. Science. 2011;331(6016):456-461.

14. Sengupta S, Peterson TR, Laplante M, Oh S, Sabatini DM. mTORC1 controls fasting-induced ketogenesis and its modulation by ageing. Nature. 2010;468(7327):1100-1104.

15. Djouadi F, Brandt JM, Weinheimer CJ, Leone TC, Gonzalez FJ, Kelly DP. The role of the peroxisome proliferator-activated receptor alpha $(\operatorname{PPAR} \alpha)$ in the control of cardiac lipid metabolism. Prostaglandins Leukot Essent Fatty Acids. 1999;60(5-6):339-343.

16. Purushotham A, Schug TT, Xu Q, Surapureddi
S, Guo X, Li X. Hepatocyte-specific deletion of SIRT1 alters fatty acid metabolism and results in hepatic steatosis and inflammation. Cell Metab. 2009;9(4):327-338.

17. Hirschey MD, et al. SIRT3 regulates mitochondrial fatty-acid oxidation by reversible enzyme deacetylation. Nature. 2010;464(7285):121-125.

18. Singh R, et al. Autophagy regulates lipid metabolism. Nature. 2009;458(7242):1131-1135.

19. Criollo A, et al. The IKK complex contributes to the induction of autophagy. $E M B O J$. 2010;29(3):619-631.

20. McCue MD. Starvation physiology: reviewing the different strategies animals use to survive a common challenge. Comp Biochem Physiol A Mol Integr Physiol. 2010;156(1):1-18.

21. Kroemer G, Marino G, Levine B. Autophagy and the integrated stress response. Mol Cell. 2010;40(2):280-293

22. Komatsu M, Kageyama S, Ichimura Y.p62/ SQSTM1/A170: physiology and pathology. Pharmacol Res. 2012;66(6):457-462.

23. Klionsky DJ, et al. Guidelines for the use and interpretation of assays for monitoring autophagy. Autophagy. 2012;8(4):445-544.

24. Mizushima N, Yamamoto A, Matsui M, Yoshimori $\mathrm{T}$, Ohsumi Y. In vivo analysis of autophagy in response to nutrient starvation using transgenic mice expressing a fluorescent autophagosome marker. Mol Biol Cell. 2004;15(3):1101-1111.

25. Rakhshandehroo M, et al. Comprehensive analysis of PPAR $\alpha$-dependent regulation of hepatic lipid metabolism by expression profiling. PPAR Res. 2007;2007:26839.

26. Rakhshandehroo M, Knoch B, Müller M, Kersten S. Peroxisome proliferator-activated receptor $\alpha$ target genes. PPAR Res. 2010;2010:612089.

27. Di Tommaso L, et al. Diagnostic value of HSP70, glypican 3, and glutamine synthetase in hepatocellular nodules in cirrhosis. Hepatology. 2007;45(3):725-734.

28. International Consensus Group for Hepatocellular Neoplasia. Pathologic diagnosis of early hepatocellular carcinoma: a report of the international consensus group for hepatocellular neoplasia. Hepatology. 2009;49(2):658-664.

29. Wunderlich FT, et al. Hepatic NF-кB essential modulator deficiency prevents obesity-induced insulin resistance but synergizes with high-fat feeding in tumorigenesis. Proc Natl Acad Sci U S A. 2008;105(4):1297-1302.

30. Xie $\mathrm{M}$, et al. A pivotal role for endogenous TGF- $\beta$-activated kinase- 1 in the LKB1/AMP-activated protein kinase energy-sensor pathway. Proc Natl Acad Sci U S A. 2006;103(46):17378-17383.

31. Menon S, et al. Chronic activation of mTOR complex 1 is sufficient to cause hepatocellular carcinoma in mice. Sci Signal. 2012;5(217):ra24.

32. Shin JH, et al. TAK1 regulates autophagic cell death by suppressing the phosphorylation of p70
S6 kinase 1. Sci Rep. 2013;3:1561.

33. Criollo A, et al. Inhibition of autophagy by TAB2 and TAB3. EMBO J. 2011;30(24):4908-4920.

34. Takaesu G, Kobayashi T, Yoshimura A. TGF $\beta-$ activated kinase 1 (TAK1)-binding proteins (TAB) 2 and 3 negatively regulate autophagy. J Biochem. 2012;151(2):157-166.

35. Shimazu T, et al. SIRT3 deacetylates mitochondrial 3-hydroxy-3-methylglutaryl CoA synthase 2 and regulates ketone body production. Cell Metab. 2010;12(6):654-661.

36. Duran A, et al. p62 is a key regulator of nutrient sensing in the mTORC1 pathway. Mol Cell. 2011;44(1):134-146.

37. Linares JF, Duran A, Yajima T, Pasparakis M, Moscat J, Diaz-Meco MT. K63 polyubiquitination and activation of mTOR by the p62-TRAF6 complex in nutrient-activated cells. Mol Cell. 2013;51(3):283-296.

38. Takamura A, et al. Autophagy-deficient mice develop multiple liver tumors. Genes Dev. 2011;25(8):795-800.

39. Inami Y, et al. Persistent activation of Nrf2 through p62 in hepatocellular carcinoma cells. JCell Biol. 2011;193(2):275-284.

40. Rosenfeldt MT, et al. p53 status determines the role of autophagy in pancreatic tumour development. Nature. 2013;504(7479):296-300.

41. Wu M, et al. Suppression of Tak1 promotes prostate tumorigenesis. Cancer Res. 2012; 72(11):2833-2843.

42. Kawahara T, Asthana S, Kneteman NM. m-TOR inhibitors: what role in liver transplantation? J Hepatol. 2011;55(6):1441-1451.

43. LoPiccolo J, Blumenthal GM, Bernstein WB, Dennis PA. Targeting the PI3K/Akt/mTOR pathway: effective combinations and clinical considerations. Drug Resist Updat. 2008;11(1-2):32-50.

44. Sato S, et al. Essential function for the kinase TAK1 in innate and adaptive immune responses. Nat Immunol. 2005;6(11):1087-1095.

45. Wu X, Sun SC. Retroviral oncoprotein Tax deregulates NF- $\kappa \mathrm{B}$ by activating Tak1 and mediating the physical association of Tak1-IKK. EMBO Rep. 2007;8(5):510-515.

46. Storch J, Zhou YX, Lagakos WS. Metabolism of apical versus basolateral sn-2-monoacylglycerol and fatty acids in rodent small intestine. J Lipid Res. 2008;49(8):1762-1769.

47. Liu Y, et al. Knockdown of acyl-CoA:diacylglycerol acyltransferase 2 with antisense oligonucleotide reduces VLDL TG and ApoB secretion in mice. Biochim Biophys Acta. 2008;1781(3):97-104.

48. Adachi M, Brenner DA. High molecular weight adiponectin inhibits proliferation of hepatic stellate cells via activation of adenosine monophosphate-activated protein kinase. Hepatology. 2008;47(2):677-685.

49. Lee JH, et al. Maintenance of metabolic homeostasis by Sestrin2 and Sestrin3. Cell Metab. 
2012;16(3):311-321.

50. Sears DD, et al. Mechanisms of human insulin resistance and thiazolidinedione-mediated insulin sensitization. Proc Natl Acad Sci U S A. 2009;106(44):18745-18750.
51. Hsiao A, Worrall DS, Olefsky JM, Subramaniam S. Variance-modeled posterior inference of microarray data: detecting gene-expression changes in 3T3-L1 adipocytes. Bioinformatics. 2004;20(17):3108-3127.
52. Hsiao A, Ideker T, Olefsky JM, Subramaniam S. VAMPIRE microarray suite: a web-based platform for the interpretation of gene expression data. Nucleic Acids Res. 2005;33(Web Server issue):W627-W632. 